\title{
Anomalous crossed Andreev reflection in mesoscopic superconducting ring hosting Majorana fermions
}

\author{
Minchul Lee, ${ }^{1}$ Heunghwan Khim, ${ }^{2}$ and Mahn-Soo $\mathrm{Choi}^{2}$ \\ ${ }^{1}$ Department of Physics, College of Applied Science, Kyung Hee University, Yongin 446-701, Korea \\ ${ }^{2}$ Department of Physics, Korea University, Seoul 136-701, Korea
}

\begin{abstract}
We investigate the Majorana physics and its effect on the electron transport in the non-topological superconductor(NS)-topological superconductor(TS) double junctions of a ring geometry. We find that, depending on the ratio between the lengths of two topologically different regions and the localization lengths of the Majorana fermions formed between them, two completely different transport mechanisms are working: perfect crossed Andreev reflection (CAR) for the short NS region and perfect normal Andreev reflection for the short TS region. The difference is explained in terms of the topologically distinct properties of subgap states in the NS-TS double junction system, which have not been revealed so far. The exotic dependence of the CAR process on the magnetic flux threading the ring is uncovered and can be used to detect the Majorana fermions.

PACS numbers: $73.63 . \mathrm{Nm}, 74.78 . \mathrm{Na}, 74.81 . \mathrm{g}, 74.45 .+\mathrm{c}$
\end{abstract}

\section{INTRODUCTION}

Solution to the Dirac equation,,${ }^{1,2}$ the first quantum theory compatible with special relativity, is complex in general, implying that to each particle there should exist an anti-particle with same mass but opposite charge. Theoretically, the Dirac equation can also have a real solution. ${ }^{3}$ The associated particle, so-called a Majorana fermion, must then be its own anti-particle and its charge neutral. Furthermore, Majorana fermions satisfy non-Abelian statistics, which can be explored for topologically protected quantum computation. ${ }^{4-6}$

Whereas Majorana fermion as an elementary particle still remains elusive with its direct observation facing formidable technical challenges, it appears to be far more abundant and experimentally accessible as an emergent quasi-particle in condensed-matter systems. ${ }^{7,8}$ Earlier, it was shown that unpaired Majorana fermions can exist localized at the ends of quantum wires with certain specific conditions, ${ }^{9}$ and recently several proposals have been put forward for realistic devices based on a semiconducting nanowire with strong spin-orbit coupling and in proximity to a superconductor. ${ }^{10-14}$ Also proposed are schemes to manipulate and braid the Majorana fermions to perform quantum gates. ${ }^{14-16}$

The zero-bias peak observed in recent experiments on $\mathrm{InSb}^{17,18}$ and InAs ${ }^{19}$ nanowire strongly suggests the existence of Majorana fermions. It may not be a decisive evidence, ${ }^{20-22}$ though, and some other evidences are worthwhile. One promising direction is to investigate the supercurrent characteristics through a Josephson junction with Majorana fermions localized at it. ${ }^{9,23-25}$

A semiconductor nanowire with strong spin-orbit coupling and in close proximity to a superconductor turns to either topologically non-trivial superconductor (hereafter called "topological superconductor" or TS) or topologically trivial conventional superconductor (to be called "non-topological superconductor" or NS). With two topologically distinct superconductors at hand, one can consider three kinds of single Josephson junctions: NS-NS, TS-TS, and NS-TS junctions. The NS-NS junction is the ordinary Josephson junction in which a Cooper pair tunnels through intermediate in- sulating or conducting medium [see Fig. 1(a)]. The supercurrent $I$ in the tunneling limit is then a sinusoidal function of the phase difference $\delta \varphi \equiv \varphi_{L}-\varphi_{R}$, being periodic with a period $2 \pi: I=I_{0} \sin \delta \varphi$. On the other hand, the TSTS junction hosts a single fermionic excitation localized at the junction [see Fig. 1(b)]. Upon the $2 \pi$ change in $\delta \varphi$, a fermionic quasi-particle is transported to the junction region and the fermion parities of the two TS regions are reversed. Another $2 \pi$ change in $\delta \varphi$ restores the fermion parities. Thus the Josephson current exhibits $4 \pi$ periodicity, unless there is any fermion-parity-breaking process. ${ }^{9,26}$ For a hybrid NS-TS junction [see Fig. 1(c)], since both sides have a gap and are topologically different, a single gapless state should exist at the boundary. ${ }^{9,14,27}$ The junction thus has a single Majorana state which is pinned at the Fermi level, irrespective of the phase difference. The supercurrent, proportional to the derivative of the Andreev bound states with respect to the phase difference [see], should then be zero. The vanishing supercurrent can be argued in another way: In the Majorana state which is its own anti-particle, the amplitudes of particle and hole excitations are the same. Further, since the particle and hole are at the same (Fermi) energy level, their group velocities have the same magnitude. Hence, their contributions to the current should cancel out each other exactly, and no current flows through the hybrid NS-TS junction.

Recently, Jiang et al. ${ }^{23}$ proposed a way to induce a supercurrent through the hybrid NS-TS junction by making a TSNS-TS double junction. In their setup, the middle NS is short
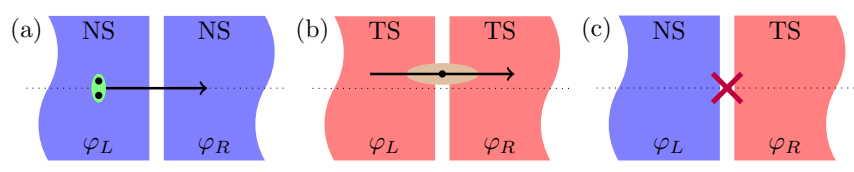

FIG. 1: (Color online) (a) NS-NS junction, with ordinary Cooperpair (double dot in ellipse) tunneling. (b) TS-TS junction, hosting a Dirac fermion (dot in ellipse) excitation at the junction. (c) NS-TS junction, hosting a single Majorana (cross) localized at the junction. $\varphi_{L}$ and $\varphi_{R}$ are the superconducting phases in the left and the right superconductors. 


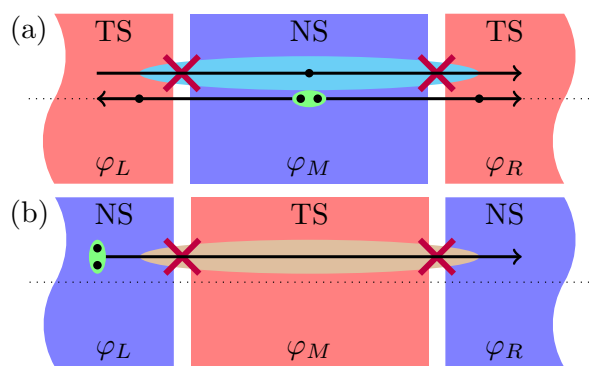

FIG. 2: (Color online) (a) TS-NS-TS double junction and (b) NSTS-NS double junction. $\varphi_{L}, \varphi_{M}$, and $\varphi_{R}$ are the superconducting phases in the left, middle, and the right superconductors.

enough that the overlap between the two Majorana states localized at both ends of the NS segment is finite. The overlap couples the two Majorana states so that their energies are lifted from the Fermi level and the vanishing current condition is no longer valid. They predicted two different mechanisms of electron tunneling [see Fig. 2(a)]: First, a single electron can tunnel from one TS to the other TS like in the TS-TS junction. Secondly, a Cooper pair in the middle NS is split, and each of two electrons from the Cooper pair tunnels into either left or right TS. The Josephson junction energy from the two tunneling mechanisms have different dependence on the superconducting phases: the Josephson energy from the former process follows that of the TS-TS junction

$$
E_{M} \cos \frac{\varphi_{L}-\varphi_{R}}{2}
$$

and the energy due to the Cooper pair splitting is given by

$$
E_{Z} \cos \left(\frac{\varphi_{L}+\varphi_{R}}{2}-\varphi_{M}\right) .
$$

It was proposed to measure unusual Shapiro steps in a nonlocal ac current in order to detect the latter tunneling mechanism.

What about a NS-TS-NS double junction [see Fig. 2(b)] with a short TS segment in the middle? Interestingly, even though it is seemingly a counter part of the TS-NS-TS double junction discussed in Ref. 23, its Majorana physics and associated supercurrent characteristics are quite different. As we will show in detail with numerically exact calculations (see Section III) and perturbation theory (see Section A), the main difference is that the energy splitting due to the overlap over the TS segment of the two Majorana states is independent of the phase difference and does not carry supercurrent. Putting another way, NS-TS-NS and TS-NS-TS double junctions have topologically different characteristics: Since NS preserves the fermion parity, it cannot accept a single electron, and the two transport mechanisms working in the TS-NS-TS double junction cannot take place. In principle, the overlap between the Majorana fermions opens a fermionic channel through the short TS so that the Cooper pair in the NS regions can tunnel through it via virtual processes as depicted in Fig. 2(b). Hence, the Josephson energy in the NS-TS-NS double junc-

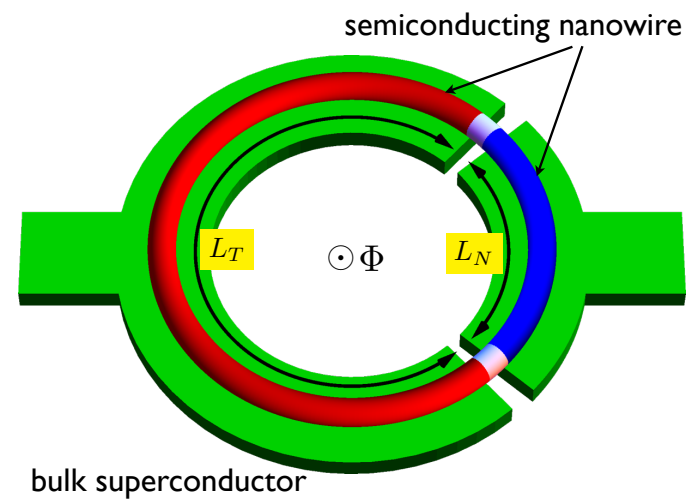

FIG. 3: (Color online) Illustration of a ring made of a semiconducting quantum wire in proximity to two $s$-wave superconductors. The underlying superconductors are interrupted by insulators so that no current can flow directly between bulk superconductors. Different gate voltages on two segments of the quantum wire define two topologically different parts whose length are $L_{T}$ and $L_{N}$, respectively. The external magnetic field $\mathbf{B}$ pierces the ring inducing the Zeeman splitting and the magnetic flux in the wire.

tion will behave like

$$
E_{C}\left[\cos \left(\varphi_{L}-\varphi_{M}\right)+\cos \left(\varphi_{M}-\varphi_{R}\right)\right]
$$

in a symmetric double junction. Since the Cooper pair tunneling demands the cotunneling processes, the magnitude of $E_{C}$ would be significantly smaller than those of $E_{M}$ and $E_{Z}$ in the counterpart setup.

In this paper we investigate the Majorana physics and the corresponding electron transport in the TS-NS double junctions of a closed ring geometry as shown in Fig. 3. This setup contains both TS-NS-TS and NS-TS-NS double junction, allowing us to study them on an equal footing. The Aharonov-Bohm phase from the threading magnetic flux and the phase difference between bulk superconductors that induce the $p$-wave superconductivity in the nanowire control the relative phases across the junctions between NS and TS. We find that the supercurrent characteristics through the ring strongly depends on the relative ratio between the segment lengths and the localization lengths of the Majorana states. For short (compared with the localization lengths of the Majorana states) NS and long TS segments (see Section III A), the supercurrent originates solely from the crossed Andreev reflection (CAR), exhibiting an unusual dependence on the magnetic flux. For short TS and long NS segments (see Section IIIB), on the contrary, the normal Andreev reflection (NAR) determines the supercurrent, whose sign can be oscillatory with the TS segment length. The difference in the supercurrent features of the two extreme cases is explained in terms of topological properties in the subgap states (see Section II C). The representative characteristics in the above two extreme cases compete with each other and show rich effects, which we study by varying the lengths of NS and TS segment (see Section III C and III D). Finally, we discuss the applicability of our setup to detect the existence of Majorana states and its physics. 
The paper is organized as follows: In Section II we describe our system of $p$-wave superconductor double junctions of the ring geometry and the method to obtain the subgap states and the corresponding supercurrent. In this section, we also discuss the elementary features of the subgap states and associated supercurrent of topological origin, which will provide the physical interpretations for the results to be presented in Section III. Section III presents and discusses the numerical results leaving the perturbative calculations in Section A. Section IV concludes the paper.

\section{MODEL AND METHOD}

\section{A. $p$-wave Superconductor Junctions}

We consider a narrow semiconductor ring in proximity to two spatially separated $s$-wave superconductors as shown in Fig. 3. The semiconductor wire forms a ring geometry with radius $R$ and circumference $L=2 \pi R .^{29}$ The underlying bulk superconductors are attached to superconducting electrodes so that the supercurrent through the ring can be measured. In the ring part, two (lower and upper) junctions at $x=x_{a}=0$ and $x=x_{b}=L_{N}$ are introduced by inserting insulating regions between superconductors. Here we assume that the insulators are thick enough so that no current can flow directly between superconductors. In the presence of the magnetic flux $\Phi$ threading the ring, the phase of the superconducting order parameter, $\varphi(x)$, depends on the position and in a proper gauge is given by $\varphi(x)=4 \pi f x / L$, where $f \equiv \Phi / \Phi_{0}$ is the dimensionless magnetic flux and $\Phi_{0} \equiv h / e$ is the flux quantum for a single electron. Apart from the magnetic-flux contribution, an additional phase difference is generated between two superconductors when a bias current is applied across the ring. Putting them all together, the overall order-parameter phase takes the form

$$
\varphi(x)=\varphi_{0}(x)+4 \pi f \frac{x}{L}
$$

with

$$
\varphi_{0}(x)= \begin{cases}\varphi_{N} & \left(x_{a}<x<x_{b}\right) \\ \varphi_{T} & \left(x_{b}<x<L\right)\end{cases}
$$

Via the proximity effect, the bulk superconductors induce an $s$-wave superconductivity on the semiconducting nanowire, on which the order parameter is given by $\Delta(x)=\Delta_{0} e^{i \varphi(x)}$.

Assuming that the semiconducting nanowire is narrow enough that only the lowest transverse mode is involved, the Hamiltonian of the superconductivity-induced wire then reads

$$
H=\oint d x\left\{\left[\psi_{\uparrow}^{\dagger}(x) \psi_{\downarrow}^{\dagger}(x)\right]\left(\frac{\Pi_{x}^{2}}{2 m}-\mu_{F}(x)+\frac{V_{Z}}{2} \sigma_{z}+\frac{\alpha}{\hbar} \frac{\left\{\boldsymbol{\sigma} \cdot \mathbf{u}(x), \Pi_{x}\right\}}{2}\right)\left[\begin{array}{l}
\psi_{\uparrow}(x) \\
\psi_{\downarrow}(x)
\end{array}\right]+\Delta(x) \psi_{\uparrow}^{\dagger}(x) \psi_{\downarrow}^{\dagger}(x)+(h . c .)\right\}
$$

with $\Pi_{x}=p_{x}-2 \pi \hbar f / L$. The field operator $\psi_{s}(x)$ describes the electronic degrees of freedom in the lowest transverse mode with spin $s=\uparrow, \downarrow$ and effective mass $m\left(m \approx 0.015 m_{e}\right.$ for $\operatorname{InSb}^{17,18}$ and $m \approx 0.03 m_{e}$ for InAs $\left.{ }^{19}\right)$. One of the key ingredient for effective $p$-wave superconductivity is the strong Rashba spin-orbit coupling, which is specified by the parameter $\alpha$ $\left(\alpha \approx 0.2 \mathrm{eV} \cdot \AA\right.$ for $\operatorname{InSb}^{17,18}$ and $\left.\operatorname{InAs}{ }^{19}\right)$ or equivalently by the spin-orbit length $\ell_{s o} \equiv \hbar^{2} / m \alpha\left(\ell_{s o} \approx 200 \mathrm{~nm}\right.$ for InSb ${ }^{17,18}$ and $\ell_{s o} \approx 127 \mathrm{~nm}$ for InAs $\left.{ }^{19}\right)$. The Rashba-induced effective magnetic field is perpendicular to the wire direction and hence varies along the wire, and $\mathbf{u}(x)=\hat{\mathbf{x}} \cos \phi(x)+\hat{\mathbf{y}} \sin \phi(x)$ is the unit vector parallel to the Rashba field at the position $x$. The inner curly brackets denote the anticommutator, and guarantees the hermiticity of $H$ in the presence of position-dependent Rashba field $\mathbf{u}(x)$. The other ingredient is the Zeeman field $V_{Z}$ perpendicular to the Rashba field, which is applied perpendicular to the ring plane. The applied magnetic field should induce a finite spin splitting but be still weak enough not to break the superconductivity $\left(B \sim 100 \mathrm{mT}^{17,18}\right) . \mu_{F}(x)$ is the position-dependent chemical potential: with $\mu_{F}(x)=\mu_{N}$ for $x_{a}<x<x_{b}$ and $\mu_{T}$ for $x_{b}<x<L$. As will be discussed below, the topological state of each region is controlled by locally tuning the chemical potential.

The model, Eq. (6) for a uniform wire (closed or open) is exactly solvable via the Bogoliubov-de Gennes (BdG) transformation in the chiral basis diagonalizing the single-particle part of the Hamiltonian. ${ }^{27}$ The two channels with chirality $\zeta= \pm$ are completely decoupled and a finite $p$-wave pairing potential between electrons with same spin in each channel is induced, whose order parameter is proportional to $\alpha \Delta_{0} / V_{Z}$ in the small momentum limit. It illustrates that the Rashba spin-orbit coupling, the Zeeman splitting, and the $s$-wave superconductivity combines together to form three indispensable ingredients to implement $p$-wave superconductor. Even though both channels exhibit the $p$-wave superconductivity, one of them (say $\zeta=+$ ) has a finite excitation gap between particle and hole bands remains finite at any value of momentum $k$, irrespective of the strength of the system parameters. On the other hand, the gap for the other channel $(\zeta=-)$ closes when the parameters are properly tuned. Hence, near the quantum phase transition point, only the $\zeta=-$ channel is relevant and one can project out the other channel by focusing on the low-energy physics.

Since our system is piece-wise uniform, we adopt the same projection on to the $\zeta=-$ channel to obtain an effective $p$ - 

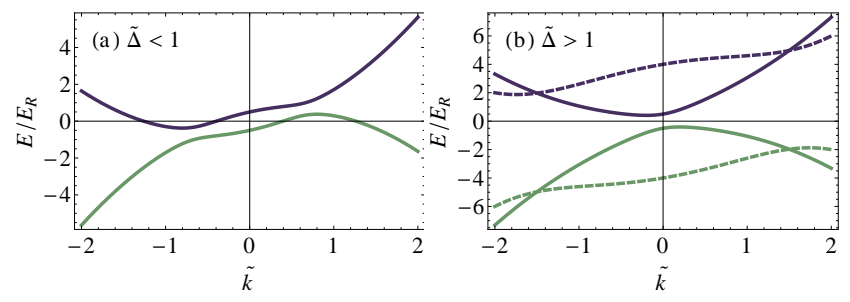

FIG. 4: (Color online) Bulk spectrum for the uniform chemical potential and superconducting phase, Eq. (11) for $p$-wave superconducting wire of ring geometry for (a) $\tilde{\Delta}<1$ and (b) $\tilde{\Delta}>1$. The case (a) is achievable only for very small rings and we focus on the case (b) in this work. In figure (b), the solid and dashed lines correspond to the bulk spectrum in the topological phase with $\tilde{\mu} \ll D$ and $\tilde{\mu} \gg D$, respectively. For the definition of $D$, see the text.

wave superconducting wire of spinless fermions:

$$
\begin{aligned}
H_{\mathrm{eff}}=\oint d x\left[\psi^{\dagger}(x)\left(\frac{\Pi_{x}^{2}}{2 m_{\mathrm{eff}}}-\mu_{\mathrm{eff}}(x)\right) \psi(x)\right. \\
\left.+\frac{\Delta_{\mathrm{eff}}(x)}{2} \psi^{\dagger}(x) \partial_{x} \psi^{\dagger}(x)+(\text { h.c. })\right]
\end{aligned}
$$

with the effective mass $m_{\mathrm{eff}}=\left(1 / m-\alpha^{2} / \hbar^{2} V_{Z}\right)^{-1}$ and the effective chemical potential $\mu_{\text {eff }}(x)=\mu_{F}(x)+V_{Z}-\Delta_{0}^{2} / 2 V_{Z}$. The induced $p$-wave order parameter

$$
\Delta_{\text {eff }}(x)=\frac{\alpha \Delta_{0}}{\hbar V_{Z}} i e^{i(\varphi(x)+\phi(x))}
$$

has two contributions to its phase: $\varphi(x)$ inherited from the phase of the order parameter of the bulk superconductors, and $\phi(x)$ from the position-dependent direction $\mathbf{u}(x)$ of the Rashba field. The corresponding BdG equation has the form

$$
i \hbar \frac{\partial}{\partial t}\left[\begin{array}{c}
\psi(x) \\
\psi^{\dagger}(x)
\end{array}\right]=H_{\mathrm{eff}}^{\mathrm{BdG}}\left[\begin{array}{c}
\psi(x) \\
\psi^{\dagger}(x)
\end{array}\right]
$$

with

$$
H_{\mathrm{eff}}^{\mathrm{BdG}}=\left[\begin{array}{cc}
\frac{\left(p_{x}-2 \pi \hbar f / L\right)^{2}}{2 m_{\mathrm{eff}}}-\mu_{\mathrm{eff}}(x) & \frac{1}{2}\left\{\Delta_{\mathrm{eff}}(x), p_{x}\right\} \\
\frac{1}{2}\left\{\Delta_{\mathrm{eff}}^{*}(x), p_{x}\right\} & -\frac{\left(p_{x}+2 \pi \hbar f / L\right)^{2}}{2 m_{\mathrm{eff}}}+\mu_{\mathrm{eff}}(x)
\end{array}\right] .
$$

Below we solve the effective model, Eqs. (7), (9), and (10) by first seeking the solution for each uniform wire segment and then matching the solutions across the junctions.

\section{B. Bulk States in a Uniform Segment}

For a uniform wire segment with $\mu_{\text {eff }}$ and $\Delta_{\text {eff }}$ constant, the bulk spectrum for an particle-like $\left(E_{+}\right)$and hole-like $\left(E_{-}\right)$ excitation are given by

$$
E_{ \pm}(\tilde{k})=E_{R}\left(\tilde{k} \pm \sqrt{\left(\tilde{k}^{2}-\tilde{\mu}\right)^{2}+\tilde{\Delta}^{2} \tilde{k}^{2}}\right)
$$

with

$$
\begin{aligned}
\tilde{k} \equiv k R, \quad E_{R} \equiv \frac{\hbar^{2}}{2 m_{\mathrm{eff}} R^{2}}, \\
\tilde{\mu} \equiv \frac{\mu_{\mathrm{eff}}}{E_{R}}-\frac{1}{4}, \quad \tilde{\Delta} \equiv \frac{\hbar\left|\Delta_{\mathrm{eff}}\right|}{E_{R} R}=\frac{2 \Delta_{0}}{V_{Z}} \frac{R}{\ell_{s o}}
\end{aligned}
$$

The spectrum becomes gapless for $\tilde{\mu}=0$, at which occurs the topological phase transition between a topological phase (T) with $\tilde{\mu}>0$ and a non-topological phase (N) with $\tilde{\mu}<0$.

The spectrum is asymmetric with respect to $\tilde{k} \rightarrow-\tilde{k}$; see Fig. 4. The variation of the Rashba field direction along the curved wire invokes the precession of electron spin, and the resulting Berry phase leads to a finite $z$ component in the spin polarization axis which is exactly opposite for clockwise $(\tilde{k}<0)$ and counterclockwise $(\tilde{k}>0)$ movers. Adding the Zeeman field, therefore, makes the magnitude of the $z$ component different for two opposite movers, introducing asymmetry between them. ${ }^{28}$ As a result, the gap between the particle and hole bands is indirect. In particular, for small $\Delta<1$, the system is metallic over the whole range of energy; see Fig. 4(a). However, such an asymmetry effect is pronounced only for very small ring $\left(R \ll \ell_{s o} V_{Z} / 2 \Delta_{0} \approx 200 \mathrm{~nm}\right)$. In our study, we therefore focus on the case with $\tilde{\Delta}>1$, where the gap $E_{\text {gap }}$ is finite and almost direct.

The bulk eigenstates corresponding to the spectrum, Eq. (11) are

$$
\begin{aligned}
& \chi_{k,+}(x)=e^{i k x}\left[\begin{array}{l}
e^{+i(\varphi / 2+(f+1 / 2) x / R)} \cos \frac{\vartheta_{k}}{2} \\
e^{-i(\varphi / 2+(f+1 / 2) x / R)} \sin \frac{\vartheta_{k}}{2}
\end{array}\right] \\
& \chi_{k,-}(x)=e^{i k x}\left[\begin{array}{l}
-e^{+i(\varphi / 2+(f+1 / 2) x / R)} \sin \frac{\vartheta_{k}}{2} \\
e^{-i(\varphi / 2+(f+1 / 2) x / R)} \cos \frac{\vartheta_{k}}{2}
\end{array}\right]
\end{aligned}
$$

with the angle $\vartheta_{k}$ defined by $\tan \vartheta_{k}=\tilde{\Delta} \tilde{k} /\left(\tilde{k}^{2}-\tilde{\mu}\right)$. The phase $\pm x / 2 R$ in the exponents originates from the variation of the Rashba field direction, $\phi(x)$, resulting in the Berry's phase $\pi$ for one cycle along the ring. As can be seen from Eq. (13), this Rashba phase always appears together with the magnetic flux $f$ in the form of $f+1 / 2$. In other words, the actual effect of the Rashba phase is to apply an additional half flux quantum $\Phi_{0} / 2$ through the ring.

\section{Topological Property of Subgap States at Junctions}

By applying a non-uniform chemical potential along the ring as given by

$$
\tilde{\mu}_{\mathrm{eff}}(x)= \begin{cases}\tilde{\mu}_{N}<0 & \left(x_{a}<x<x_{b}\right) \\ \tilde{\mu}_{T}>0 & \left(x_{b}<x<L\right)\end{cases}
$$

the two segments become topologically different superconductors, and a localized Majorana state is formed at each interface $x=x_{a, b} \cdot{ }^{9,14}$ Before obtaining exact subgap states in the non-uniform configuration (see Sec. II D), we examine the topological structure of the Majorana states localized at the junctions. Here we focus on the case of isolated Majorana 
states and disregard the interaction between them. For further simplicity, we turn off the magnetic flux $(f=0)$. Without the interaction, the energy of the Majorana state is zero, and by seeking zero-energy solution in Eqs. (11) and (13), we obtain four complex wave vectors

$$
k_{\eta \nu}^{\ell}=\eta\left[(-1)^{\nu} k_{r}+i / \lambda_{\ell \nu}\right]
$$

and the corresponding wave functions

$$
\chi_{\eta, \nu}^{\ell}(x)=e^{i k_{\eta \nu}^{\ell} x}\left[\begin{array}{l}
e^{+i\left(\varphi_{\ell} / 2+x / 2 R\right)} e^{+i \gamma_{\eta \nu}^{\ell} / 2} \\
e^{-i\left(\varphi_{\ell} / 2+x / 2 R\right)} e^{-i \gamma_{\eta \nu}^{\ell} / 2}
\end{array}\right]
$$

for each region $\ell=T, N$. Here the index $\eta= \pm$ denotes the decay direction of the wave function tail $(\eta=+(-)$ state decays in the positive (negative) direction) and $\nu=1,2$ distinguishes different Majorana modes with different localization lengths $\left(\lambda_{\ell 1} \geq \lambda_{\ell 2}\right)$. The wave vectors $k_{\eta \nu}^{\ell}$ are complex and their imaginary parts determine the localization length of the Majorana states. In the NS region $\left(\tilde{\mu}_{N}<0\right)$, all wave vectors are pure imaginary $\left(k_{r}=0\right)$ and the localization lengths are given by

$$
\lambda_{N \nu}=\frac{R}{\sqrt{D+\left|\tilde{\mu}_{N}\right|}+(-1)^{\nu} \sqrt{D}}
$$

with $D \equiv\left(\tilde{\Delta}^{2}-1\right) / 4$. In the TS region $\left(\tilde{\mu}_{T}>0\right)$, the real part is given by

$$
k_{r}= \begin{cases}0 & \left(0<\tilde{\mu}_{T}<D\right) \\ \frac{\sqrt{\tilde{\mu}_{T}-D}}{R} & \left(\tilde{\mu}_{T}>D\right)\end{cases}
$$

and the localization length by

$$
\lambda_{T \nu}=\left\{\begin{array}{ll}
\frac{R}{\sqrt{D}+(-1)^{\nu} \sqrt{D-\tilde{\mu}_{T}}} & \left(0<\tilde{\mu}_{T}<D\right) \\
\frac{R}{\sqrt{D}} & \left(\tilde{\mu}_{T}>D\right)
\end{array} .\right.
$$

The relative phase difference $\gamma_{\eta \nu}^{N}=\eta(-1)^{\nu} \gamma$ in the NS region depends on both $\eta$ and $\nu$, where the angle $\gamma$ has been defined by

$$
e^{i \gamma} \equiv-\frac{1}{\tilde{\Delta}}+i \sqrt{1-\frac{1}{\tilde{\Delta}^{2}}}
$$

but $\gamma_{\eta \nu}^{T}=\eta \gamma$ in the TS region does not depend on $\nu$. This difference leads to intriguing topological properties as we discuss below. The wave functions $\Psi_{i}(x)$ for Majorana states localized at $x=x_{i}(i=a, b)$ are then given by linear superpositions of the eigenstates, Eq. (16); refer their explicit forms to Eq. (A1). The coefficients for eigenstates are determined by the matching condition at each junction:

$$
\Psi_{i}\left(x_{i}^{+}\right)=\Psi_{i}\left(x_{i}^{-}\right), \quad v_{x} \Psi_{i}\left(x_{i}^{+}\right)=v_{x} \Psi_{i}\left(x_{i}^{-}\right)
$$

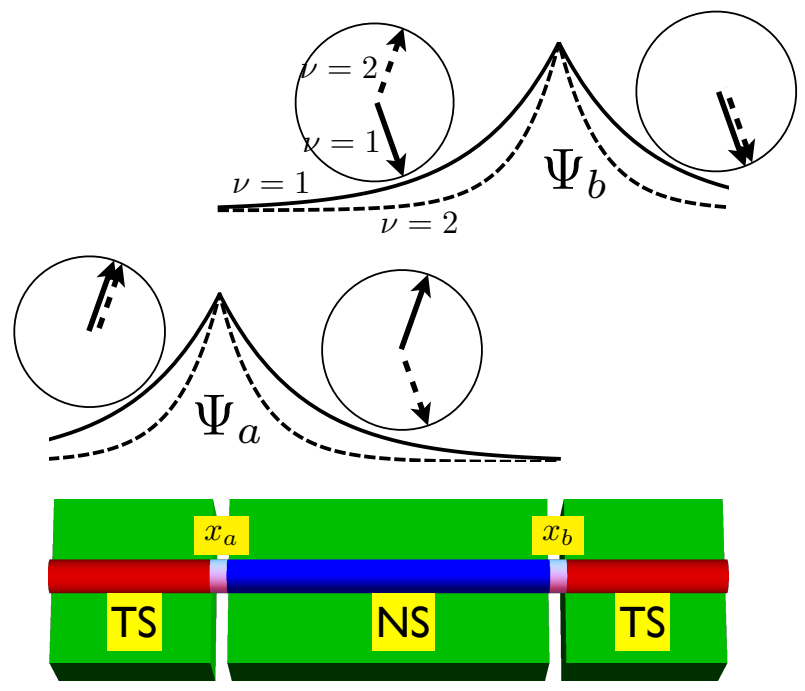

FIG. 5: (Color online) A schematic representation of the subgap states, $\Psi_{a}$ and $\Psi_{b}$, at the TS-NS $\left(x_{a}\right)$ and NS-TS $\left(x_{b}\right)$ junction interface, respectively. The curves depict the spatial distributions of the wave functions, and the arrows in circles the pseudo-spin polarizations in the particle-hole basis. The solid and dotted curves/arrows correspond to $\nu=1$ and 2 Majorana mode, respectively. Here no superconducting phase difference $(\delta \varphi=0)$ is applied for simplicity. Note that for finite length of the NS segment, the wave function $\Psi_{a}$ should be matched with $\Psi_{b}$ properly at $x=x_{b}$, and $\Psi_{b}$ with $\Psi_{a}$ at $x=x_{a}$. When matching, $\nu=1$ mode undergoes a pseudo-spin rotation by angle $2 \gamma$, while $\nu=2$ mode does not.

where $v_{x}$ is the velocity operator along the wire

$$
v_{x}=\left[\begin{array}{cc}
-i R \partial_{x}-f & \frac{\tilde{\Delta}}{2} e^{+i\left(\varphi(x)+(2 f+1) \frac{x}{R}\right)} \\
\frac{\tilde{\Delta}}{2} e^{-i\left(\varphi(x)+(2 f+1) \frac{x}{R}\right)} & i R \partial_{x}-f
\end{array}\right] .
$$

Note that $x_{a}^{-}=L$.

In order to clarify the topological difference between the wave functions, Eq. (16) of the NS and TS regions, we regard the wave functions as spinors in the pseudo-spin up ( $\uparrow$ ) and down $(\downarrow)$ basis in the particle-hole (or so-called Nambu) space, and examine their pseudo-spin polarization directions. Figure 5 shows the pseudo-spin polarization of the eigenstates localized at the TS-NS and NS-TS junctions for $\delta \varphi=0$. We see the clear difference between relative pseudo-spin polarizations in two topologically different regions. In the NS region, the two evanescent modes $(\nu=1,2)$ localized at the same end form an angle $2 \gamma(\pi / 2<\gamma<\pi)$, while they are parallel to each other in the TS region: For an infinite-curvature ring $(\tilde{\Delta} \gg 1), \gamma \approx \pi / 2$ so the two modes are polarized in the opposite direction. The phase difference $\delta \varphi=\varphi_{N}-\varphi_{T}$ leads to the misalignment between the polarization axes for the two regions. $^{30}$

This topological difference leads to two important consequences which are experimentally detectable. First, the overlap between Majorana fermions has different nature according to whether they are coupled through the NS or the TS regions. For example, in the TS region, the pseudo-spins of two modes are always aligned and rotate in the same way so they are always in phase, which is the main reason why the overlap of 
Majorana states through the TS region is almost a constant independent of phases. On the other hand, the two modes in the NS region are not aligned so that their amplitudes depend on the superconducting phases and the magnetic flux. In the following sections, we will see a stark contrast in the properties of the supercurrents in the two cases.

Second, the two modes acquire different phases while they travel through the NS region; see Fig. 5. While the $\nu=1$ mode in the left TS region tunnels to the NS region without a rotation, it has to rotate by $2 \gamma$ to match with the $\nu=1$ mode in the right TS region. This rotation should be reflected in the overlap matrix element between $\Psi_{a}$ and $\Psi_{b}$. However, the $\nu=2$ mode rotates in the left TS-NS junction, while it is then already aligned with the $\nu=2$ mode in the right TS region. The pseudo-spin rotation at the left junction just contribute to an overall phase of $\Psi_{a}$ so that it does not affect the $\Psi_{a}-\Psi_{b}$ overlap matrix element. Hence, the phase difference $2 \gamma$ between two modes arises. Note that this additional phase does not take place in the case of the crossed Andreev reflection (Cooper pair splitting) where two electrons in the middle NS region go in the opposite directions. In this case the pseudo-spin rotates in the opposite directions for oppositemoving electrons so that the phases are canceled out. Hence, this pseudo-spin rotation in the particle-hole space severely affects the relative amplitudes of currents due to the singleelectron tunneling and the crossed Andreev reflection through the TS-NS-TS junctions.

\section{Subgap States and Supercurrent in a Closed Ring}

For finite-size segments between two junctions, as in our system of ring geometry (see Fig. 3), the wave functions of two localized Majorana modes have a finite overlap, which gives rise to finite energies $\pm E_{A}$ of subgap eigenstates with $\left|E_{A}\right| \leq E_{\text {gap }}=\min \left(E_{\text {gap }}^{T}, E_{\text {gap }}^{N}\right)$ where $E_{\text {gap }}^{\ell}$ is the gap in each region $(\ell=T, N)$. The overlap $E_{A}$ depends exponentially on the ratio of the segment length $L_{\ell}(\ell=N, T)$ to the localization lengths $\lambda_{\ell \nu}$ of the Majorana states. The effective low-energy Hamiltonian can be then written as

$$
H_{M}=E_{A}\left(2 d^{\dagger} d-1\right)
$$

where $d=\left(\gamma_{a}+i \gamma_{b}\right) / \sqrt{2}$ is the fermionic operator from the Majorana fermion operators $\gamma_{a, b}$. The subgap eigenstates are then labeled as $|0\rangle$ and $|1\rangle \equiv d^{\dagger}|0\rangle$. The supercurrent corresponding to the eigenstate is then calculated by taking the derivative of the energy: ${ }^{31}$

$$
I=\frac{2 e}{\hbar} \frac{\partial H_{M}}{\partial \delta \varphi} .
$$

We determine the exact subgap energy $E_{A}$ by solving the BdG equation, Eq. (9) in each region and matching the solutions across the interfaces at $x=x_{a}$ and $x_{b}$ imposing the boundary conditions analogous to Eq. (21). Explicitly, one has to solve self-consistently $E_{A}=E_{+}(\tilde{k})\left[E_{A}=E_{-}(\tilde{k})\right.$ gives identical results due to the particle-hole symmetry] and the boundary condition

$$
\Psi\left(x_{i}^{+}\right)=\Psi\left(x_{i}^{-}\right), \quad v_{x} \Psi\left(x_{i}^{+}\right)=v_{x} \Psi\left(x_{i}^{-}\right)
$$

for $i=a, b$ and

$$
\Psi(x)=\sum_{\eta \nu} \begin{cases}c_{\eta \nu}^{N} \chi_{k_{\eta \nu}^{N},+}^{N}(x) & \left(x_{a}<x<x_{b}\right) \\ c_{\eta \nu}^{T} \chi_{k_{\eta \nu}^{T},+}^{T}(x) & \left(x_{b}<x<L\right)\end{cases}
$$

Here $k_{\eta \nu}^{\ell}$ are four solutions of $E_{A}=E_{+}(\tilde{k})$ with $\tilde{\mu}=\tilde{\mu}_{\ell}$ and $c_{\eta \nu}^{\ell}$ the coefficient for each mode. In the following section, the self-consistent equations are numerically solved to obtain and examine the energy $E_{A}$ as a function of the magnetic flux $f$ and the phase difference $\delta \varphi$ for given parameters.

Throughout the paper, we choose $R \approx 300 \mathrm{~nm}, m \approx$ $0.015 m_{e}\left(m_{e}\right.$ is the bare electron mass), $\alpha \approx 2 \times 10^{-11} \mathrm{eVm}$, and $\Delta_{0} \approx V_{Z} \approx 300 \mu \mathrm{eV}$, which are suitable for realistic samples. They correspond to $E_{R} \approx 20 \mu \mathrm{eV}$ and $\tilde{\Delta} \approx 3$ in the effective model. The value of $\tilde{\mu}_{\ell}$ can be varied by the gate voltage.

\section{RESULTS AND DISCUSSIONS}

In this section we present the subgap eigenenergy $E_{A}$ and the supercurrent $I$ by using the method described in Sec. II. First, we consider two extreme cases where either the NS (Section III A) or TS (Section III B) region is short compared with the localization lengths of the Majorana states, and show that the two cases exhibit distinct behaviors in subgap energy and supercurrent. We then examine the evolution of one case to the other by changing continuously the relative length between two regions, and the length dependence of each supercurrent is discussed (Section III C). Finally, we study the small ring case (Section III D) where both the behaviors should arise simultaneously.

\section{A. Short NS Region $\left(L_{N} \sim \lambda_{N 1}, L_{T} \gg \lambda_{T 1}\right)$}

First, we consider the case in which the NS region is short and the TS region is very long: $L_{N} \sim \lambda_{N 1}$ and $L_{T} \gg \lambda_{T 1}$. Figures 6, 7, and 8 present our numerical results for the subgap eigenenergy $E_{A}$ as functions of $\delta \varphi$ and $f$. We find that these results fit well to the expression

$$
E_{A} \approx E_{M} \cos \left(2 \pi f+\gamma_{M}\right)+E_{Z} \cos \left(\delta \varphi-2 \pi f+\gamma_{Z}\right) .
$$

Figures 7 and 8 show that for $L_{N} \gtrsim \lambda_{N 1}$, the coefficients $E_{M}$ and $E_{Z}$ and the phase shifts $\gamma_{M} \approx \pi-2 \gamma$ and $\gamma_{Z} \approx 0$ are in a good agreement with the approximate results (dashed line) calculated perturbatively in Sec. A. In a large ring $(\tilde{\Delta} \gg 1)$ with a short NS segment $\left(L_{N} \sim \lambda_{N 1}\right)$, the perturbation theory suggests a simpler expression of the coefficients and the phase shifts: $\gamma_{M} \approx \gamma_{Z} \approx 0$ and

$$
\begin{gathered}
E_{M} \approx E_{R} \frac{\epsilon_{1}}{N_{0}}\left(e^{-L_{N} / \lambda_{N 1}}+e^{-L_{N} / \lambda_{N 2}}\right) \\
E_{Z} \approx E_{R} \frac{\epsilon_{1}}{N_{0}}\left(e^{-L_{N} / \lambda_{N 1}}-e^{-L_{N} / \lambda_{N 2}}\right)
\end{gathered}
$$




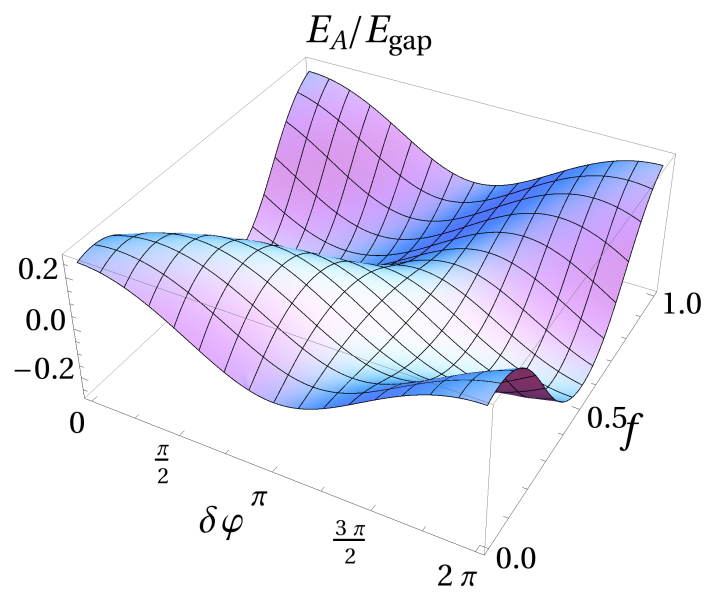

FIG. 6: (Color online) Subgap energy for the state $|1\rangle$ as a function of $\delta \varphi$ and $f$ for the short NS region and long TS region: $L_{N} / \lambda_{N 1} \approx$ 1.55 and $L_{T} / \lambda_{T 1} \approx 7.11$. Here we have used $\tilde{\Delta}=3, \tilde{\mu}_{N}=-5$, $\tilde{\mu}_{T}=5$, and $E_{\text {gap }} \approx 79 \mu \mathrm{eV}$.

Refer the definition of $\epsilon_{1}$ and $N_{0}$ to Eqs. (A13) and (A17).

The subgap expression, Eq. (27) is consistent with that of Jiang et al. ${ }^{23}$ [see Eqs. (1) and (2)]. To see this, substitute the superconducting phases as follows:

$$
\varphi_{L} \rightarrow \varphi_{T}, \quad \varphi_{M} \rightarrow \varphi_{N}, \quad \varphi_{R} \rightarrow \varphi_{T}+4 \pi(f+1 / 2) .
$$

Note that the phase shift $4 \pi(f+1 / 2)$ in $\varphi_{R}$ is the phase acquired by a Cooper pair circling around the ring in the presence of the magnetic flux and the Rashba field. Then, the single-electron tunneling term becomes

$$
E_{M}^{\mathrm{Jiang}} \cos \frac{\varphi_{L}-\varphi_{R}}{2} \rightarrow-E_{M}^{\mathrm{Jiang}} \cos 2 \pi f
$$

corresponding to the $E_{M}$ term in Eq. (27), and the Cooper pair splitting term becomes

$E_{Z}^{\mathrm{Jiang}} \cos \left(\frac{\varphi_{L}+\varphi_{R}}{2}-\varphi_{M}\right) \rightarrow-E_{Z}^{\mathrm{Jiang}} \cos (\delta \varphi-2 \pi f)$.

corresponding to the $E_{Z}$ term in Eq. (27). The sign change is ascribed to the Rashba phase which adds additional phase $\pi$ upon circling around the ring.

The $E_{M}$-term comes from the circulation of a single electron around the ring. In fact, the phase $2 \pi f$ is exactly the magnetic phase acquired by a single electron enclosing the magnetic flux $f$. The additional phase $\gamma_{M}$ arises from the asymmetry between clockwise and counterclockwise movers due to the finite curvature of the ring, as discussed in Sec. II B. Equation (28a) shows that the contributions from two modes are simply additive. As discussed in Sec. II C, the $\nu=1$ mode acquires the phase $2 \gamma \approx \pi$ with respect to the $\nu=2$ mode, which leads to a sign difference between them. On the other hand, the diagonal component of the velocity operator in Eq. (22) suggests that the supercurrent measures the pseudospin current. Since the pseudo-spins of the two modes in the

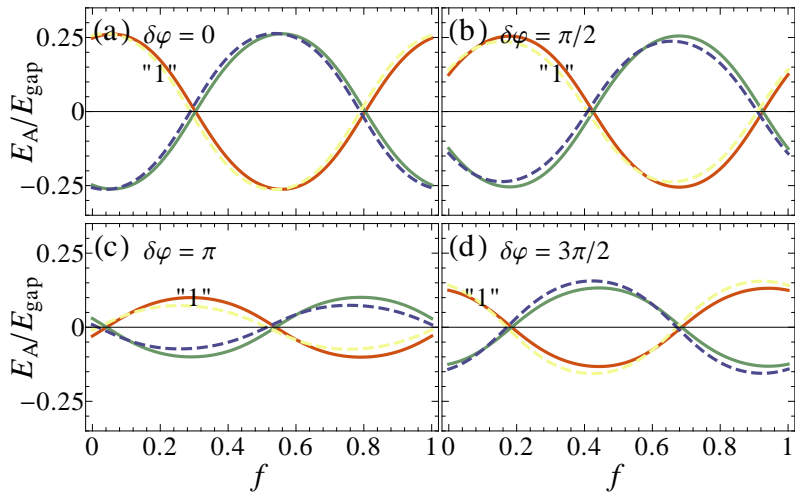

FIG. 7: (Color online) Subgap energies as a function of $f$ for fixed values of $\delta \varphi$ as annotated. The energy which corresponds to the state $|1\rangle$ is marked by "1". Solid and dashed lines correspond to the exact and perturbative energies, respectively. We have used the same values for parameters as used in Fig. 6.

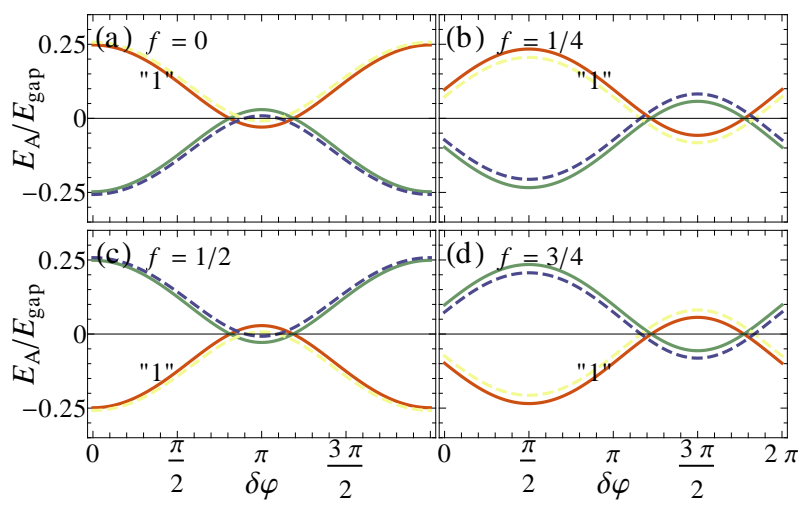

FIG. 8: (Color online) Subgap energies as a function of $\delta \varphi$ for fixed values of $f$ as annotated. Same notations and parameters are used as in Fig. 7.

NS region are opposite to each other (when $\gamma \approx \pi / 2$ ), their contribution to the supercurrent is opposite in sign. Hence, gathering two sign changes, there is no sign difference between the two modes.

The $E_{Z}$-term is due to the Cooper pair tunneling between TS and NS region, accompanying the splitting of the Cooper pair. The two electrons of a Cooper pair tunnel between two regions through the two TS-NS boundaries, respectively. In other words, the crossed Andreev reflection takes place without any normal Andreev reflection accompanied. This perfect CAR is due to the interesting characteristic of the TS-NS junction as discussed in Sec. I: no Cooper pair can tunnel directly across a single TS-NS junction.

Here three remarks are worthwhile concerning the CAR process involved in the $E_{Z}$-term. (i) As seen in Eqs. (27) and (31), the CAR process acquires the phase $\delta \varphi-2 \pi f$. The phase $\delta \varphi$ is obviously due to the tunneling of a Cooper pair between two different superconductors. The appearance of the phase $2 \pi f$ is interesting because it is identical to that by the circulation of a single electron. It indicates that the splitting and the recombination of the Cooper pair should take place at the 


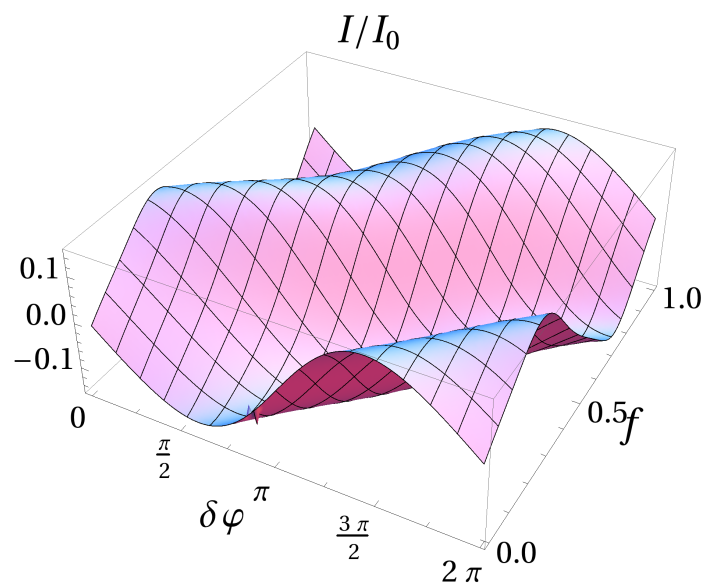

FIG. 9: (Color online) Supercurrent for the state $|1\rangle$ as a function of $\delta \varphi$ and $f$ for the short non-topological region and long topological region. We have used the same values for parameters as used in Fig. 6. Here $I_{0} \equiv \frac{2 e}{\hbar} E_{\text {gap }}$.

same TS-NS boundary so that only one of two electrons split moves around the ring before the recombination, resulting in the phase $2 \pi f$. The recombined Cooper pair at one of the boundaries then flows into the bulk superconductor, not being affected by the magnetic flux any more. It is consistent with the fact that the Majorana fermions are localized at the boundaries. This dependence on the magnetic flux is the evidence that the CAR is realized via the Majorana fermions.

(ii) Unlike in the $E_{M}$-term above, no extra pseudo-spin rotation between two modes (see Section IIC) accompanying tunneling across NS-TS junctions takes place, while the pseudo-spin currents of the two modes are opposite in sign. Hence, Eq. (28b) exhibits the negative combination between two modes.

(iii) As a consequence of the effect (ii), the $E_{Z}$-term depends non-monotonically on the length $L_{N}$ of the NS segment. The exponential factor $e^{-L_{N} / \lambda_{N \nu}}$ in both the $E_{M^{-}}$ and $E_{Z}$-term implies that the finite overlap between Majorana fermions is indispensable to observe these processes. It is also known that the CAR process can happen substantially only over lengths shorter than the size of the Cooper pair (i.e., the superconducting coherence length). Based on both, one may naively expect that the CAR process (and hence $E_{Z}$ ) get stronger with decreasing $L_{N}$. However, the tunneling processes through two modes $\nu=1,2$ gives opposite contributions as shown in the above (ii), due to the topological characteristic of the subgap states. Therefore the CAR process becomes weaker if the NS segment is too small: $E_{Z}$ increases as $L_{N}$ decreases until $L_{N} \geq \lambda_{N 2}$, but decreases again if $L_{N}$ decreases further and gets smaller than $\lambda_{N 2}\left(\leq \lambda_{N 1}\right)$. This non-monotonic dependence of the $E_{Z}$-term on $L_{N}$ will indeed be demonstrated explicitly in Figures 18(a) and 19(a), Sec. III C.

Since in both the $E_{M}$ - and $E_{Z}$-term it is a single electron, not a Cooper pair, that circulates around the ring, the periodicity of the subgap energy $E_{A}$ with respect to the magnetic flux $f$ is 1 , not $1 / 2$ as in the normal superconductor ring, which

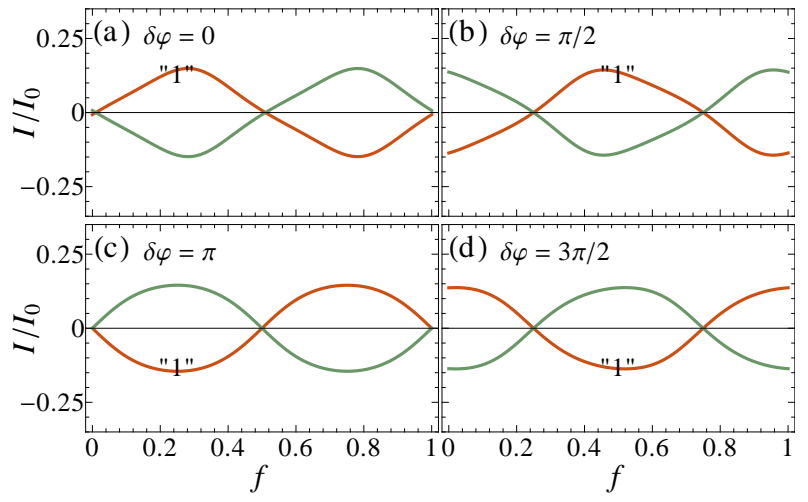

FIG. 10: (Color online) Supercurrent as a function of $f$ for fixed values of $\delta \varphi$ as annotated. Same notations and parameters are used as in Fig. 7.

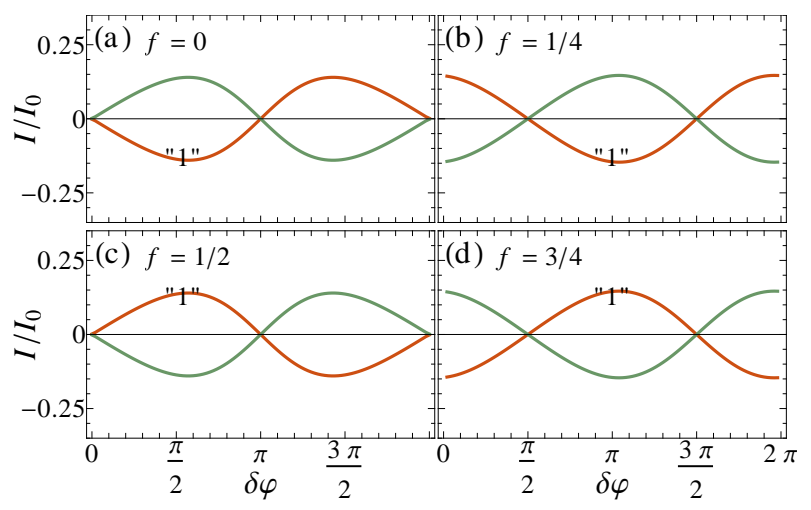

FIG. 11: (Color online) Supercurrent as a function of $\delta \varphi$ for fixed values of $f$ as annotated. Same notations and parameters are used as in Fig. 7.

is clearly revealed in Fig. 7. The $f=1$ periodicity is protected as long as no fermion-parity breaking mechanism is introduced into the system; If the parity breaking is present, the periodicity would be reduced to $1 / 2$.

Figures 9, 10, and 11 show the corresponding supercurrent, assuming that the fermion parity is preserved. From Eqs. (23), (24), and (27), the supercurrent is obtained as

$$
I \approx\left(2 d^{\dagger} d-1\right) \frac{2 e}{\hbar} E_{Z} \sin (\delta \varphi-2 \pi f) .
$$

The supercurrent obtained numerically is not exactly sinusoidal since it includes the contribution from higher-order processes: In fact, the coefficients $E_{Z}$ and $E_{M}$ are also functions of $\delta \varphi$ through the normalization constants $N_{a}$ and $N_{b}$ [see Sec. A]. As a matter of fact, only the $E_{Z}$-term contributes to the supercurrent: the $E_{M}$-term does not involve the transport of the Cooper pair. The current also exhibits the $f=1$ periodicity, which is the fingerprint of the Majorana fermions. In the presence of the parity breaking, the $f=1$ periodicity may fade away. However, one can still detect the existence of the Majorana fermion by examining the response of the supercurrent with respect to the variation of both $\delta \varphi$ and $f$. In the following section, we will compare the supercurrents due to 
the crossed and normal Andreev reflections and discuss how to distinguish them. One thing to be noted here is that in the CAR process the role of the magnetic flux $f$ is shifting the current by $2 \pi f$ without modulating the amplitude of the current.

Finally, we would like to note that all the properties of the subgap energy and the supercurrent are independent of the length of the TS region as long as it is sufficiently larger than the size of the Majorana fermions. It is in contrast to the high dependence of the $E_{Z}$-term on the relative length between the NS segment length and the Cooper pair size. It indicates that the Majorana fermion state in the TS region is highly nonlocal. In other words, this $L_{T}$-independence reflects that the correlation length and the size of the Cooper pair in the TS are almost infinite as long as the coherence is preserved.

\section{B. Short TS Region $\left(L_{N} \gg \lambda_{N 1}, L_{T} \sim \lambda_{T 1}\right)$}

Now we consider the opposite case in which the TS region is short and the NS region long: $L_{T} \sim \lambda_{T 1}$ and $L_{N} \gg \lambda_{N 1}$. Interestingly, in this case the physics of the Majorana fermions is completely different as shown in Figures 12, 13, and 14. In this regime, we obtain the following empirical expression for the subgap energy:

$$
E_{A} \approx E_{0}+E_{C}[\cos \delta \varphi+\cos (4 \pi f-\delta \varphi)]
$$

Figures 13, and 14 show that our exact and perturbative results match well with each other. The simpler expressions for the coefficients $E_{0}$ and $E_{C}$ are at hand in the large-ring limit and

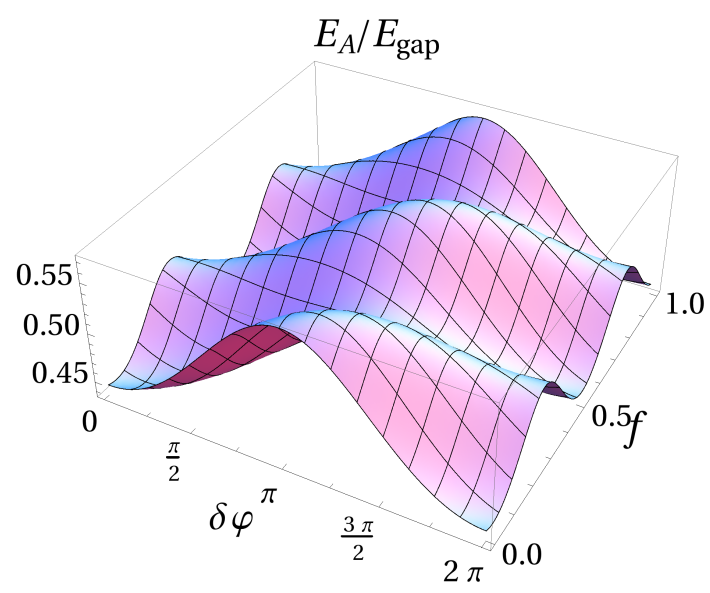

FIG. 12: (Color online) Subgap energy for the state $|1\rangle$ as a function of $\delta \varphi$ and $f$ for the long non-topological region and short topological region: $L_{N} / \lambda_{N 1} \approx 6.96$ and $L_{T} / \lambda_{T 1} \approx 0.89$. Here we have used $\tilde{\Delta}=3, \tilde{\mu}_{N}=-5, \tilde{\mu}_{T}=5$, and $E_{\text {gap }} \approx 79 \mu \mathrm{eV}$.

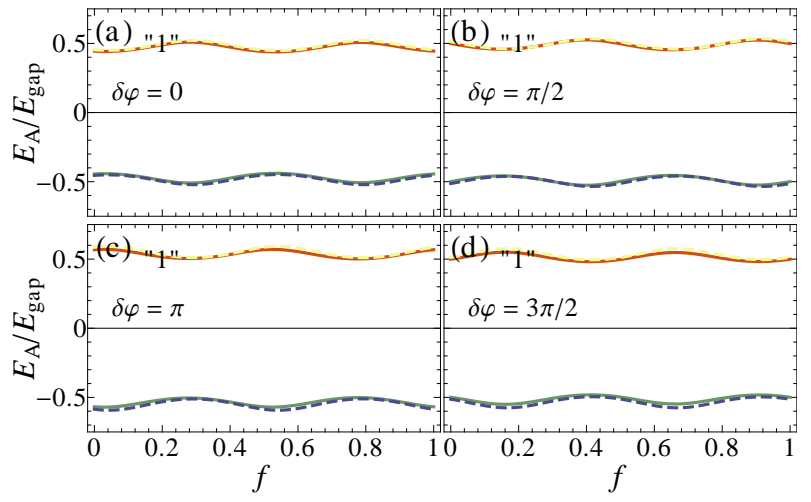

FIG. 13: (Color online) Subgap energies as a function of $f$ for fixed values of $\delta \varphi$ as annotated. We have used the same values for parameters as used in Fig. 12.

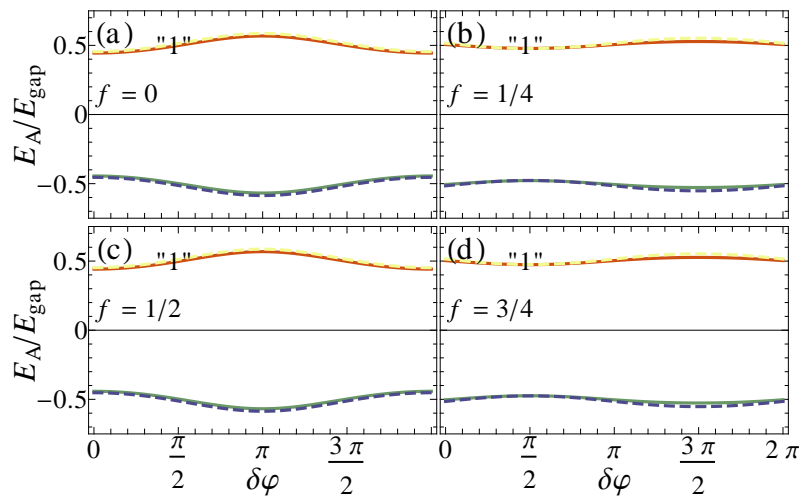

FIG. 14: (Color online) Subgap energies as a function of $\delta \varphi$ for fixed values of $f$ as annotated. Same notations and parameters are used as in Fig. 13.

for $L_{T} / \lambda_{T 1} \gtrsim 1$ :

$$
\begin{aligned}
& E_{0} \approx \frac{E_{R}}{N_{0}}\left\{\begin{array}{c}
\left(\epsilon_{1}+\epsilon_{2}\right) e^{-L_{T} / \lambda_{T 1}} \\
+\left(\epsilon_{1}-\epsilon_{2}\right) e^{-L_{T} / \lambda_{T 2}}, \\
2 \epsilon_{1} e^{-L_{T} / \lambda_{T 1}} \cos k_{r} L_{T} \\
+2 \epsilon_{2} e^{-L_{T} / \lambda_{T 1}} \sin k_{r} L_{T}, \quad \tilde{\mu}_{T}>D
\end{array}\right. \\
& E_{C} \approx \frac{\sqrt{D}}{2 \tilde{\mu}_{N} N_{0}} E_{0} .
\end{aligned}
$$

See Eqs. (A13) and (A17) for the definition of $\epsilon_{1,2}$ and $N_{0}$.

The overlap between the Majorana fermions through the TS region gives rise to a finite constant level splitting, $E_{0}$-term which is independent of $f$ and $\delta \varphi$. Note that such a constant term is missing in the former case where the overlap happens in the NS region. This is attributed to the topological difference between subgap states in TS and NS regions: The pseudo-spin directions of the two subgap states $(\nu=1,2)$ in the TS region are parallel to each other (see Figure 5). Technically, the coefficients in Eqs. (A3) and (A4) in the TS region do not depend on the phases $f$ and $\delta \varphi$. The constant splitting $E_{0}$ increases as the TS segment length decreases, eventually 
reaching the band gap $E_{\text {gap }}$ at $L_{T} \rightarrow 0$. Because of this constant splitting, no crossing between the subgap states at the Fermi level takes place.

The phase-dependent term, $E_{C}$-term is identical to that of a SQUID made of two normal Josephson junctions threaded by a magnetic flux $f$, in which the phase differences in the two junctions are $\delta \varphi$ and $4 \pi f-\delta \varphi$, respectively. The $E_{C}$-term can be directly inferred by substituting the superconducting phases in Eq. (3) according to the same rule, Eq. (29) as used in the short-NS-region case.

$$
\begin{gathered}
E_{C}\left[\cos \left(\varphi_{L}-\varphi_{\mathrm{M}}\right)+\cos \left(\varphi_{\mathrm{M}}-\varphi_{R}\right)\right] \\
\rightarrow E_{C}[\cos \delta \varphi+\cos (4 \pi f-\delta \varphi)]
\end{gathered}
$$

This dependence on $\delta \varphi$ and $f$ confirms our prediction discussed in Sec. I that it is a Cooper pair that tunnels through the NS-TS junctions if the Majorana fermions are coupled via the NS region [see Fig. 2(b)]. The overlap between Majorana fermions opens a channel at $E_{A} \neq 0$. The Cooper pair then circulates around the ring via the successive Andreev reflection in each junction. Hence the periodicity of the subgap energy $E_{A}$ with respect to the magnetic flux $f$ is $1 / 2$ as can be seen in Fig. 13. Note that the Rashba phase does not affect the $E_{C}$-term since it gives rise to a phase $4 \pi \times 1 / 2=2 \pi$. Namely, the Rashba phase acquired by a Cooper pair is twice larger than that of a single electron.

Figures 15, 16, and 17 show the corresponding supercurrent. From Eqs. (23), (24), and (33), the supercurrent is obtained as

$$
\begin{aligned}
I & \approx\left(1-2 d^{\dagger} d\right) \frac{2 e}{\hbar} E_{C}[\sin \delta \varphi+\sin (\delta \varphi-4 \pi f)] \\
& =\left(1-2 d^{\dagger} d\right) \frac{4 e}{\hbar} E_{C} \cos 2 \pi f \sin (\delta \varphi-2 \pi f) .
\end{aligned}
$$

The approximate expression, Eq. (37) shows some discrepancy from the numerically exact results. It is because the latter includes the contributions from higher-order processes: The

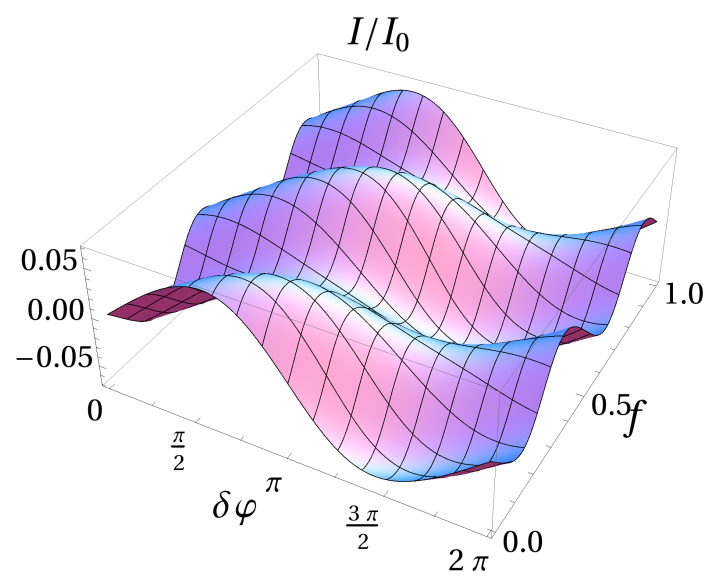

FIG. 15: (Color online) Supercurrent for the state $|1\rangle$ as a function of $\delta \varphi$ and $f$ for the long non-topological region and short topological region. We have used the same values for parameters as used in Fig. 12. Here $I_{0} \equiv \frac{2 e}{\hbar} E_{\text {gap }}$.

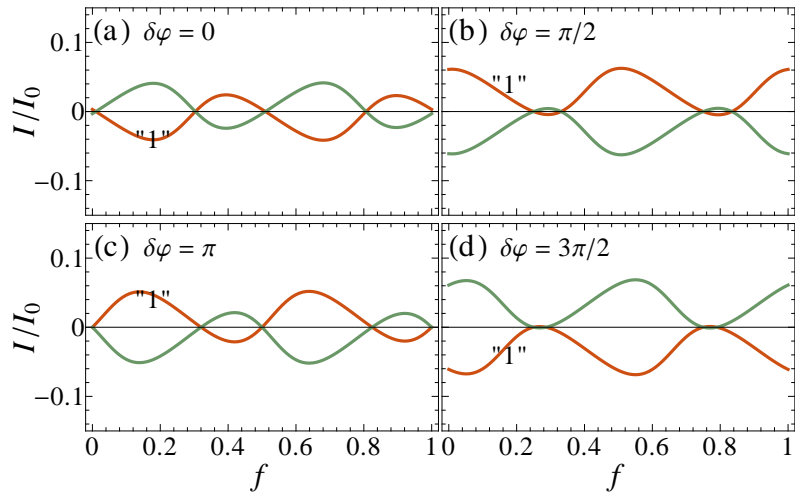

FIG. 16: (Color online) Supercurrent as a function of $f$ for fixed values of $\delta \varphi$ as annotated. Same notations and parameters are used as in Fig. 13.

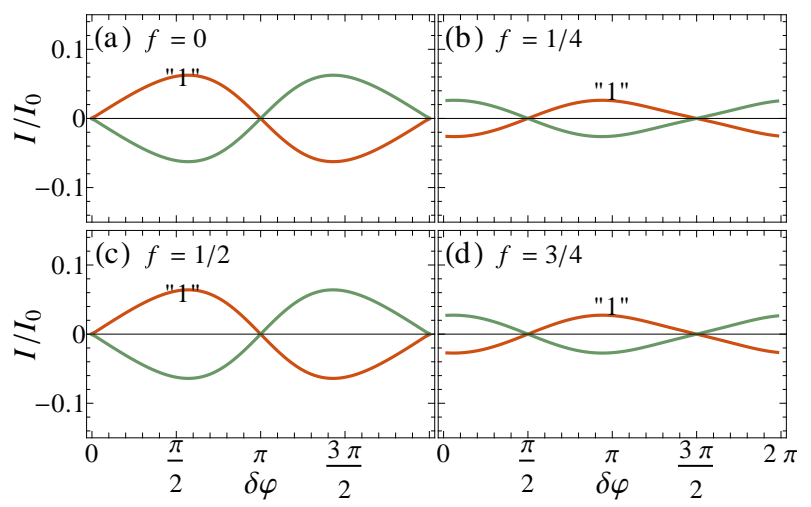

FIG. 17: (Color online) Supercurrent as a function of $\delta \varphi$ for fixed values of $f$ as annotated. Same notations and parameters are used as in Fig. 13.

current does not vanish at $f=1 / 4$ and $3 / 4$. The $f=1 / 2$ periodicity is well shown in Fig. 16. By comparing Eq. (32) and (37), one can notice that while in both cases the magnetic flux $f$ shifts the current by $2 \pi f$, it also modulates clearly the amplitude of the current in the NAR process with the weighting factor $\cos 2 \pi f$ : see the variation of the current amplitudes with respect to $f$ in Fig. 17. Hence, apart from the periodicity with respect to $f$, the modulation of the current can be used to detect the CAR phenomena due to the Majorana fermions.

Figures 15, 16, and 17 show the corresponding supercurrent. From Eqs. (23), (24), and (33), the supercurrent is obtained as

$$
\begin{aligned}
I & \approx\left(1-2 d^{\dagger} d\right) \frac{2 e}{\hbar} E_{C}[\sin \delta \varphi+\sin (\delta \varphi-4 \pi f)] \\
& =\left(1-2 d^{\dagger} d\right) \frac{4 e}{\hbar} E_{C} \cos 2 \pi f \sin (\delta \varphi-2 \pi f) .
\end{aligned}
$$

The numerically calculated supercurrent does not exactly behave as Eq. (37) since it includes the contributions from higher-order processes: The current does not vanish at $f=$ $1 / 4$ and $3 / 4$. The $f=1 / 2$ periodicity is well shown in Fig. 16. By comparing Eq. (32) and (37), one can notice that while in both cases the magnetic flux $f$ shifts the current by $2 \pi f$, 
it also modulates clearly the amplitude of the current in the NAR process with the weighting factor $\cos 2 \pi f$ : see the variation of the current amplitudes with respect to $f$ in Fig. 17. Hence, apart from the periodicity with respect to $f$, the modulation of the current can be used to detect the CAR phenomena due to the Majorana fermions.

One more interesting property of the $E_{0}$ - and $E_{C}$-term, in comparison with the $E_{Z}$ - and $E_{M}$-term, is that their sign can be controlled by tuning the TS segment length and/or the chemical potential $\tilde{\mu}_{T}$ in the deep topological region $\left(\tilde{\mu}_{T}>\right.$ D) [see Eq. (34b)]. Apart from the exponentially decreasing envelop part, the $E_{0}$ and $E_{C}$-terms are oscillatory with $k_{r} L_{T}$. This is owing to the oscillatory behavior of the Majorana wave function in the deep topological phase due to the finite real part of the wave vectors, $k_{r}$ [see Eq. (18)]. This oscillatory feature is peculiar in that it cannot be observed in the usual normal superconductor SQUID hosting no Majorana fermions. It provides an electronic way to change the sign of the supercurrent.

\section{Length Dependence}

Having understood the transport mechanisms in two extreme cases, we now examine intermediate cases varying the relative lengths of the NS and TS segment. Figure 18 displays the dependence of the coefficients $\left(E_{0}, E_{C}, E_{M}\right.$, and $\left.E_{Z}\right)$ and the phase shifts $\left(\gamma_{M}\right.$ and $\left.\gamma_{Z}\right)$ on the segment lengths $L_{N}$ and $L_{T}$ with $L=L_{N}+L_{T}$ fixed. Obviously, the coefficients $E_{M}$ and $E_{Z}$, exhibiting the exponential dependence on $L_{N}$, are finite for $L_{N} \lesssim \lambda_{N 1}$, and $E_{0}$ and $E_{C}$ are so for $L_{T} \lesssim \lambda_{T 1}$. For $L_{N} \gg \lambda_{N 1}$ and $L_{T} \gg \lambda_{T 1}$, all the coefficients are vanishingly small, since the overlap between the Majorana fermions is negligible and no transport through the NS-TS junction is possible. The coefficients $E_{M}$ and $E_{0}$ approach the energy gap $E_{\text {gap }}$ as $L_{N} \rightarrow 0$ and $L_{T} \rightarrow 0$, respectively. In these limits, the Majorana fermions are strongly bound so that they become completely fermionic. On the other hand, the coefficients $E_{Z}$ and $E_{C}$, responsible for the tunneling of Cooper pairs through the ring, remain relatively small as the segments length decreases. $E_{Z}$ goes to zero as $L_{N} \rightarrow 0$, which is due to the topological nature of the subgap states as discussed in Sec. III A. $E_{C}$ is small compared with $E_{M}$ and $E_{Z}$ because the Cooper pair tunneling, via the normal Andreev reflection, is higher-order process: Note that the $E_{M}$ and $E_{Z}$-terms originates from a single electron circulation around the ring.

As pointed out in the previous section, Fig. 18(a) shows sign-changing oscillatory behaviors of the coefficients $E_{0}$ and $E_{C}$ when the TS region is in the deep topological phase, $\tilde{\mu}_{T}(=5)>D(=2)$. The period of the oscillation is $2 \pi / k_{r}$ as expected from the sinusoidal dependence in Eq. (34a). In deeper topological phase $\left(\tilde{\mu}_{T} \gg D\right)$, the period becomes shorter [see Fig. 19(a)], resulting in more number of oscillations of $E_{0}$ and $E_{c}$ before they are suppressed. Hence, the sign of the supercurrent can be controlled not only by tuning the TS segment length $L_{T}$ with $k_{r}$ fixed but also by changing the period $2 \pi / k_{r}$ with $L_{T}$ fixed. The latter control can be done by tuning the chemical potential $\tilde{\mu}_{T}$ : Note that $k_{r}=\sqrt{\tilde{\mu}_{T}-D}$.
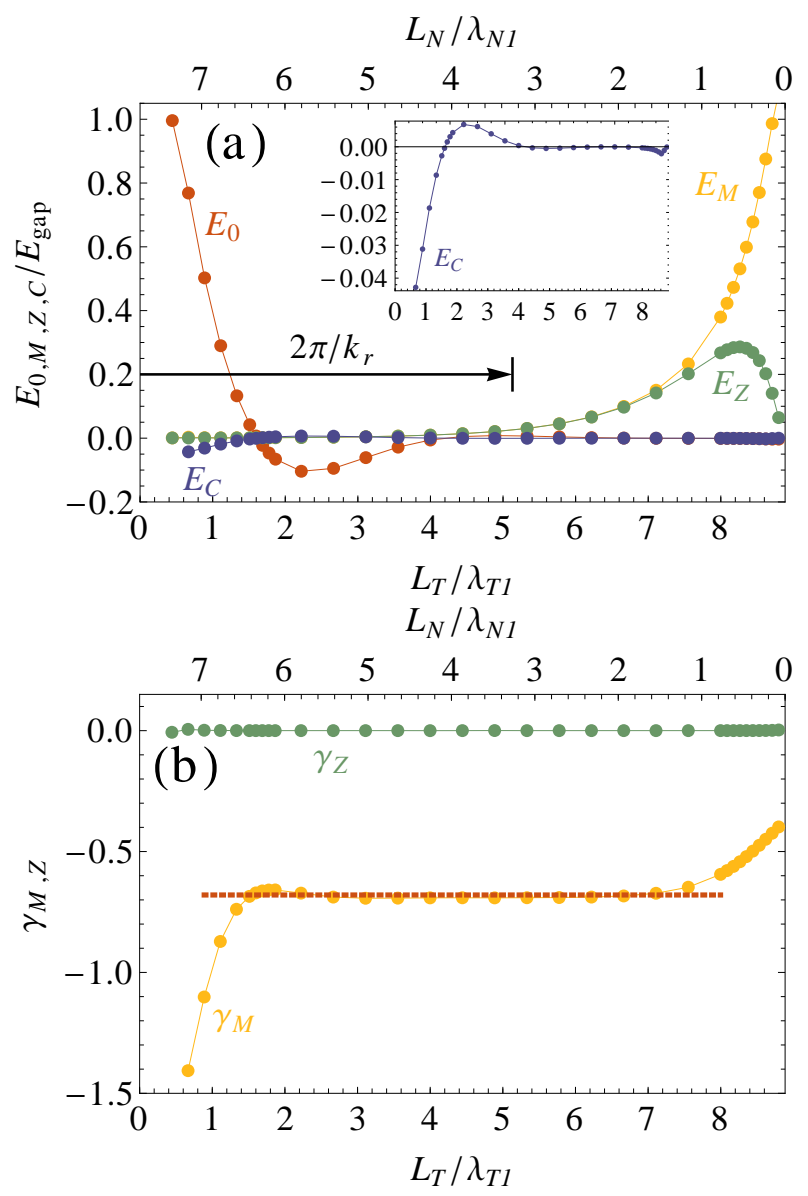

FIG. 18: (Color online) (a) The coefficients $E_{0}, E_{C}, E_{M}$, and $E_{Z}$ as functions of $L_{N}$ or $L_{T}=L-L_{N}$ with $L=L_{N}+L_{T}$ fixed. The inset shows the enlarged view of $E_{C}$. (b) The phase shifts $\gamma_{M}$ and $\gamma_{Z}$ as functions of $L_{N}$ or $L_{T}$. The dashed line correspond to the perturbative prediction for $\gamma_{M}, \pi-2 \gamma$. Here we have used $\tilde{\Delta}=3$, $\tilde{\mu}_{N}=-5$, and $\tilde{\mu}_{T}=5$.

If the TS region is in the weak topological phase, no oscillation is observed [see Fig. 19(b)] and the monotonic dependence of the coefficients $E_{0}$ and $E_{C}$ on $L_{T}$ is observed.

Finally, we examine the length dependence of the phase shifts shown in Fig. 18(b). The phase $\gamma_{Z}$ is found to be zero, irrespective of the segment length. It implies that the curvature of the ring does not affect the transport due to the CAR process. In contrast, the phase $\gamma_{M}$ is finite for all the length. In the case of $L_{N}>\lambda_{N 1}$ and $L_{T}>\lambda_{T 1}$, where the perturbation is valid, the phase is given by $\pi-2 \gamma$, the phase shift for the $\nu=1$ mode [see Eq. (A10)] for $\gamma^{\prime} \approx 0$. For $L_{N}<\lambda_{N 1}$, both $\nu=1,2$ modes are contributing so that the phase $\gamma_{M}$ becomes length-dependent. The phase $\gamma_{M}$ becomes negligible only when the size of the ring is sufficiently large: in this case $\tilde{\Delta} \gg 1$ and $\gamma \approx \pi / 2$ [see Eq. (20)]. The phase shift $\gamma_{M}$ comes from the finite curvature of the ring and the resultant phase shift of the Majorana states. Our results show that this phase appears only in the $E_{M}$-term, not in the $E_{Z}$ and, more importantly, $E_{C}$ terms. Hence, the existence of the finite phase shift $\gamma_{M}$ can be used for the evidence of the Majorana 

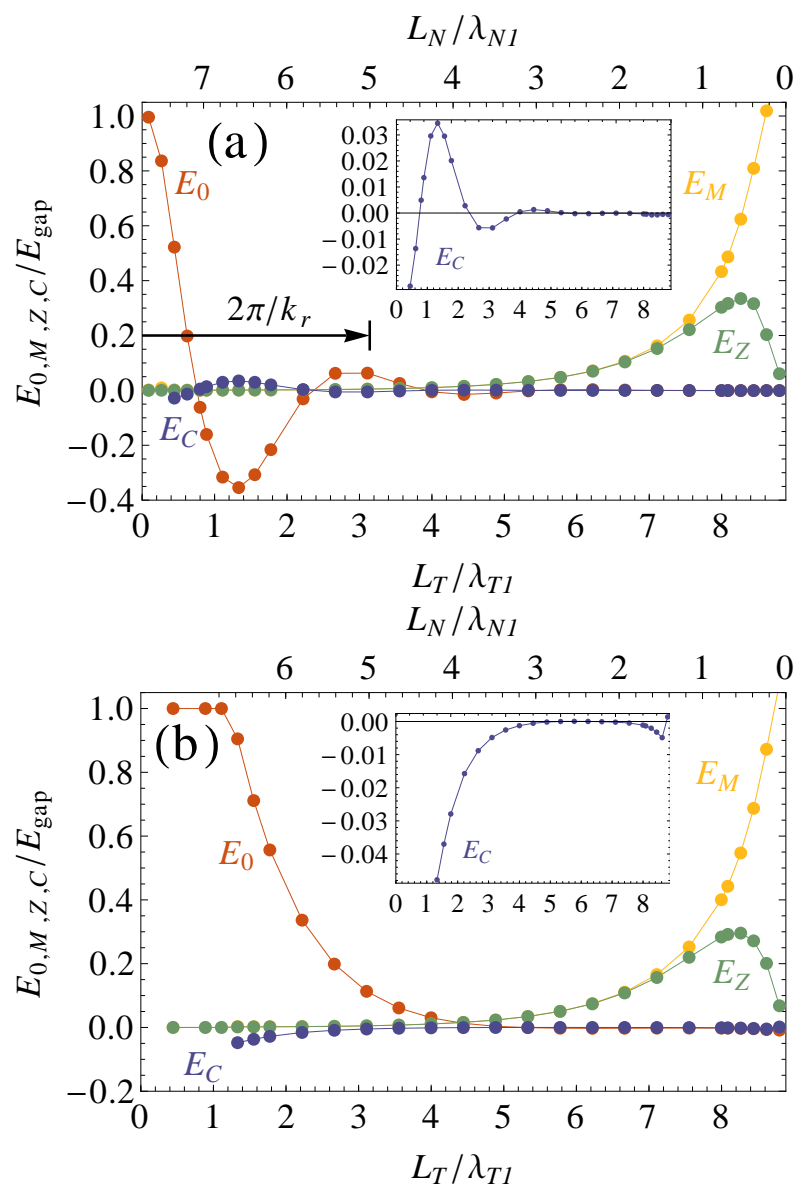

FIG. 19: (Color online) The coefficients $E_{0}, E_{C}, E_{M}$, and $E_{Z}$ as functions of $L_{N}$ or $L_{T}=L-L_{N}$ with $L=L_{N}+L_{T}$ fixed. The inset shows the enlarged view of $E_{C}$. The parameters used are $\tilde{\Delta}=3, \tilde{\mu}_{N}=-5$, and $\tilde{\mu}_{T}=10$ (deep topological phase) [(a)] and 2.5 (weak topological phase) [(b)].

fermions.

\section{Small Rings $\left(L_{N} \sim \lambda_{N 1}, L_{T} \sim \lambda_{T 1}\right)$}

Up to now, we have considered the cases in which the ring is large enough that only one of CAR and NAR processes is operative. However, if the ring is small or the localization length of the Majorana fermion is comparable to the circumference of the ring, both processes can coexist. The general form of the subgap energy is then given by

$$
\begin{aligned}
E_{A} \approx & E_{0}+E_{C}[\cos \delta \varphi+\cos (4 \pi f-\delta \varphi)] \\
& +E_{M} \cos \left(2 \pi f+\gamma_{M}\right) \\
& +E_{Z} \cos \left(\delta \varphi-2 \pi f+\gamma_{Z}\right) .
\end{aligned}
$$

In Figs. 20 and 21, we present the subgap energy in the case where both $L_{N}$ and $L_{T}$ are comparable to the Majorana fermion size, $L_{N} / \lambda_{N 1} \sim L_{T} / \lambda_{T 1} \sim 2$. In this case we ob-

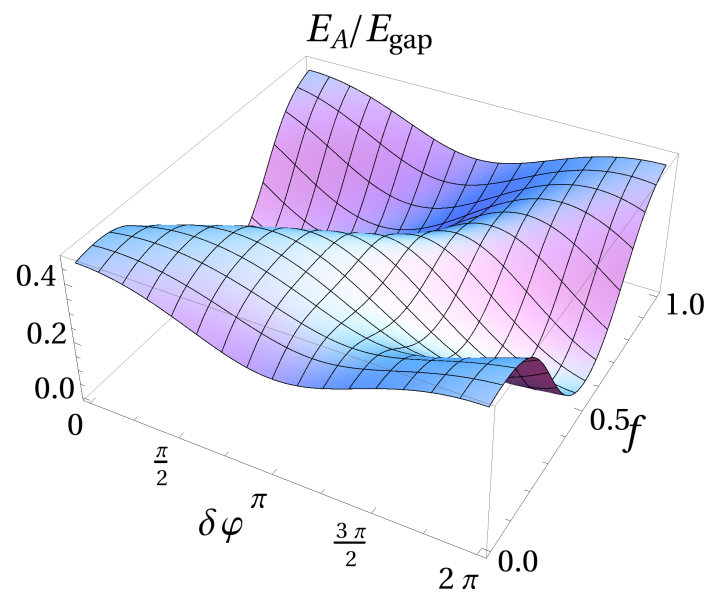

FIG. 20: (Color online) Subgap energy for the state $|1\rangle$ as a function of $\delta \varphi$ and $f$ for the short non-topological region and short topological region: $L_{N} / \lambda_{N 1} \approx 1.84$ and $L_{T} / \lambda_{T 1} \approx 1.81$. Here we have used $\tilde{\Delta}=3, \tilde{\mu}_{N}=-2, \tilde{\mu}_{T}=1.3$, and $E_{\text {gap }} \approx 25 \mu \mathrm{eV}$.

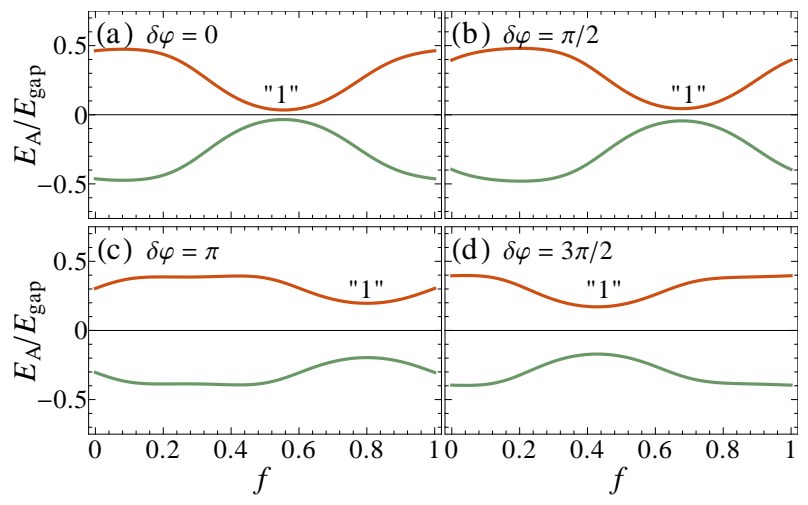

FIG. 21: (Color online) Subgap energies as a function of $f$ for fixed values of $\delta \varphi$ as annotated. The energy which corresponds to the state $|1\rangle$ is marked by " 1 ". We have used the same values for parameters as used in Fig. 20.

tain

$$
\begin{aligned}
& \frac{E_{0}}{E_{\text {gap }}} \approx 0.30, \quad \frac{E_{C}}{E_{\text {gap }}} \approx-0.02, \\
& \frac{E_{M}}{E_{\text {gap }}} \approx 0.13, \quad \frac{E_{Z}}{E_{\text {gap }}} \approx 0.12, \quad \gamma_{M} \approx-0.23, \quad \gamma_{Z} \approx 0 .
\end{aligned}
$$

While the $E_{C}$-term is still small due to its nature of high-order processes, the other terms are comparable. Since the $E_{C}$-term is negligible, the supercurrent through the ring is entirely due to the CAR process. The most intriguing point here is that the $f=1$ periodicity is protected even if there is fermion parity breaking. Figure 21 shows that there is no crossing between the state $|0\rangle$ and $|1\rangle$. The constant $E_{0}$-term, larger than the other terms, makes a big energy separation between $|0\rangle$ and $|1\rangle$ states so that they are not coupled even if there is fermion parity breaking. This kind of protection of $f=1$ periodicity was also noticed in Ref. 25. In our system, this protection not only guarantees to observe the $f=1$ periodicity but also 
provides us with a way to observe the perfect CAR process without other deterioration.

\section{CONCLUSION}

We have considered a system of TS-NS double junctions in ring geometry to investigate its supercurrent characteristics associated with the underlying topological properties and Majorana subgap states localized at the junctions. The system allows us to study on an equal footing TS-NS-TS and NSTS-NS double junction, which turn out to have topologically distinct supercurrent characteristics. In this setup, the relative phases across the junctions are controlled by the AharonovBohm phase from the threading magnetic flux as well as the phase difference between bulk the superconductors that induce the $p$-wave superconductivity in the nanowire.

We have found that TS-NS-TS and NS-TS-NS double junction, seemingly counterpart of each other, have substantially different supercurrent characteristics due to the topological properties of their subgap states. In our ring geometry containing both types of double junction, the supercurrent characteristics depend strongly on the ratios of the wire segment lengths and the localization lengths of the Majorana states. For short (compared with the localization lengths of the Majorana states) NS and long TS segment (Section III A), the supercurrent originates solely from the crossed Andreev reflection, exhibiting an unusual dependence on the magnetic flux. For short TS and long NS segment (Section III B), on the contrary, the normal Andreev reflection (NAR) determines the supercurrent, whose sign can be oscillatory with the TS segment length. The difference in the supercurrent features of the two extreme cases is explained in terms of topological properties in the subgap states (Section IIC). The representative characteristics in the above two extreme cases compete with each other and show rich effects, which we study by varying the lengths of NS and TS segment (Section III C and III D).

\section{Acknowledgments}

This work was supported by the National Research Foundation of Korea (NRF) grants funded by the Korea MEST (Nos. 2011-0030790, 2011-0012494, and 2010-0025880).

\section{Appendix A: Perturbative Approach}

In order to obtain an analytical expression for the subgap energy $E_{A}$ as a function of $\delta \varphi$ and $f$, we take a perturbative approach, performing a similar calculation used in Refs. 23 and 25. First we neglect the interaction between two Majorana states, each of which is localized at the interface, through the TS and NS regions. In our ring geometry, it is done by applying the boundary conditions only at one of the boundaries, see Eq. (21). Suppose that $\Psi_{a}(x)$ and $\Psi_{b}(x)$ are the unperturbed Majorana wave functions localized at $x=x_{a}$ and $x=b$, respectively. Then, $\Psi_{b}(x)$ is the zero-energy eigenstate of the
Hamiltonian, Eq. (10) in the region $0<x<L$ including the boundary $x=x_{b}$, but not $x=x_{a}$. Therefore, $\Psi_{b}(x)$ satisfies the boundary condition at $x=b$ only. Similarly, $\Psi_{a}(x)$ is defined in the region $L_{N}<x<L+L_{N}$ and satisfies the boundary conditions at $x=x_{a}$ only. The wave functions are then given by linear combinations of the zero-energy eigenstates in Eq. (16), whose coefficients are determined via the boundary conditions, Eq. (21). Explicitly, the normalized wave functions for $\Psi_{i}(x)(i=a, b)$ are

$$
\Psi_{i}(x)= \begin{cases}\Psi_{i N}(x), & x_{a}<x<x_{b} \\ \Psi_{i T}(x), & x_{b}<x<L\end{cases}
$$

with

$$
\begin{aligned}
& \Psi_{a N}(x)=\sum_{\nu} \frac{c_{a N \nu}}{\sqrt{N_{a}}} \chi_{+, \nu}^{N}(x) \\
& \Psi_{a T}(x)=\sum_{\nu} \frac{c_{a T \nu}}{\sqrt{N_{a}}} e^{\left(i \nu k_{r}-1 / \lambda_{N \nu}\right) L} \chi_{-, \nu}^{T}(x) \\
& \Psi_{b N}(x)=\sum_{\nu} \frac{c_{b N \nu}}{\sqrt{N_{b}}} e^{-L_{N} / \lambda_{N \nu}} \chi_{-, \nu}^{N}(x) \\
& \Psi_{b T}(x)=\sum_{\nu} \frac{c_{b T \nu}}{\sqrt{N_{b}}} e^{-\left(i \nu k_{r}-1 / \lambda_{N \nu}\right) L_{N}} \chi_{+, \nu}^{T}(x) .
\end{aligned}
$$

Note here that the additional exponential factors have been inserted to make the coefficients of order one at the localization center. The coefficients are

$$
\begin{aligned}
& c_{a N 1}=\frac{\sin (\gamma-2 \pi(f+1 / 2)+\delta \varphi / 2)}{\sin \gamma} \\
& c_{a N 2}=e^{i \gamma} \frac{\sin (2 \pi(f+1 / 2)-\delta \varphi / 2)}{\sin \gamma} \\
& c_{a T 1}=\frac{1}{2}\left(1+\sqrt{\frac{D-\tilde{\mu}_{N}}{D-\tilde{\mu}_{T}}}\right) \\
& c_{a T 2}=\frac{1}{2}\left(1-\sqrt{\frac{D-\tilde{\mu}_{N}}{D-\tilde{\mu}_{T}}}\right)
\end{aligned}
$$

and

$$
\begin{aligned}
& c_{b N 1}=\frac{\sin (\gamma-\delta \varphi / 2)}{\sin \gamma} \\
& c_{b N 2}=e^{-i \gamma} \frac{\sin (\delta \varphi / 2)}{\sin \gamma} \\
& c_{b T 1}=\frac{1}{2}\left(1+\sqrt{\frac{D-\tilde{\mu}_{N}}{D-\tilde{\mu}_{T}}}\right) \\
& c_{b T 2}=\frac{1}{2}\left(1-\sqrt{\frac{D-\tilde{\mu}_{N}}{D-\tilde{\mu}_{T}}}\right)
\end{aligned}
$$

The normalization constants are given by

$$
\begin{aligned}
N_{i}=\frac{1}{R} \sum_{\nu} & {\left[\frac{\left|c_{i N \nu}\right|^{2}}{\lambda_{N \nu}^{-1}}+2 \cos \gamma \frac{\left|c_{i N \nu} c_{i N \bar{\nu}}\right|}{\lambda_{N 1}^{-1}+\lambda_{N 2}^{-1}}\right.} \\
+ & \left.\frac{\left|c_{i T \nu}\right|^{2}}{\lambda_{T \nu}^{-1}}+\frac{2 c_{i T \nu}^{*} c_{i T \bar{\nu}}}{2(-1)^{\nu} i k_{r}+\lambda_{T 1}^{-1}+\lambda_{T 2}^{-1}}\right] .
\end{aligned}
$$


Here the normalization constants are obtained up to the leading order in the small factor $e^{-L / \lambda_{\ell \nu}}$, which is consistent with our perturbation. Note that the Rashba phase appear explicitly in the coefficients $c_{a N \nu}$ in the form of $f+1 / 2$, as discussed in Sec. II B.

The effective Hamiltonian projected to the Majorana subspace is then represented as

$$
H_{\mathrm{M}}=\left[\begin{array}{cc}
\left\langle\Psi_{a}\left|H_{\mathrm{eff}}^{\mathrm{BdG}}\right| \Psi_{a}\right\rangle & \left\langle\Psi_{a}\left|H_{\mathrm{eff}}^{\mathrm{BdG}}\right| \Psi_{b}\right\rangle \\
\left\langle\Psi_{b}\left|H_{\mathrm{eff}}^{\mathrm{BdG}}\right| \Psi_{a}\right\rangle & \left\langle\Psi_{b}\left|H_{\mathrm{eff}}^{\mathrm{BdG}}\right| \Psi_{b}\right\rangle
\end{array}\right]
$$

Since $\Psi_{i}(x)$ are not the eigenstates of the full Hamiltonian $H_{\text {eff }}^{\mathrm{BdG}}$, the diagonal terms does not vanish. However, we ignore them since they are proportional to the square of the exponential factor $e^{-L / \lambda_{\ell \nu}}$ and much smaller than the offdiagonal terms. The formal expression for the off-diagonal terms are

$$
\begin{aligned}
H_{M, a b}=i\{ & \Psi_{a}^{\dagger}\left(x_{a}\right)\left(v_{\phi} \Psi_{b}\left(x_{a}^{-}\right)-v_{\phi} \Psi_{b}\left(x_{a}^{+}\right)\right) \\
& \left.+\left[v_{\phi} \Psi_{a}\left(x_{a}\right)\right]^{\dagger}\left(\Psi_{b}\left(x_{a}^{-}\right)-\Psi_{b}\left(x_{a}^{+}\right)\right)\right\} \\
H_{M, b a}=-i & \left\{\Psi_{b}^{\dagger}\left(x_{b}\right)\left(v_{\phi} \Psi_{a}\left(x_{b}^{+}\right)-v_{\phi} \Psi_{a}\left(x_{b}^{-}\right)\right)\right. \\
+ & \left.+\left[v_{\phi} \Psi_{b}\left(x_{b}\right)\right]^{\dagger}\left(\Psi_{a}\left(x_{b}^{+}\right)-\Psi_{a}\left(x_{b}^{-}\right)\right)\right\} .
\end{aligned}
$$

Since $\Psi_{a}(x)$ and $\Psi_{b}(x)$ are not orthogonal to each other, the effective Hamiltonian is not necessarily hermitian, $H_{M, a b} \neq$ $H_{M, b a}^{*}$. The subgap energy is then obtained as

$$
E_{A}= \pm \sqrt{H_{M, a b} H_{M, b a}} .
$$

Explicit and tedious calculations lead to

$$
\begin{aligned}
& H_{M, a b}=+i E_{R} \frac{e^{-i \gamma}}{\sqrt{N_{a} N_{b}}} \sum_{\ell \nu} e^{-L_{\ell} / \lambda_{\ell \nu}} h_{\ell \nu}^{-} \\
& H_{M, b a}=-i E_{R} \frac{e^{+i \gamma}}{\sqrt{N_{a} N_{b}}} \sum_{\ell \nu} e^{-L_{\ell} / \lambda_{\ell \nu}} h_{\ell \nu}^{+}
\end{aligned}
$$

with

$$
h_{N \nu}^{ \pm}=\frac{(-1)^{\nu} \epsilon_{1}}{\sin \gamma}\left[\cos \left(2 \pi f+\gamma^{\prime}-\zeta_{\nu}\right)-\cos \left(\delta \varphi-2 \pi f \pm \gamma^{\prime}\right)\right]
$$

with $\zeta_{1}=2 \gamma$ and $\zeta_{2}=0$ and

$$
h_{T \nu}^{ \pm}=\epsilon_{1} \sin \left(\gamma-\gamma^{\prime}\right)-(-1)^{\nu}\left(\epsilon_{2} \sin \gamma-\epsilon_{3} \cos \gamma\right)
$$

for $0<\tilde{\mu}_{T}<D$ and

$$
\begin{aligned}
\sum_{\nu} h_{T \nu}^{ \pm}= & 2 \epsilon_{1} \sin \left(\gamma-\gamma^{\prime}\right) \cos k_{r} L_{T} \\
& +2\left(\epsilon_{2} \sin \gamma-\epsilon_{3} \cos \gamma\right) \sin k_{r} L_{T}
\end{aligned}
$$

for $D<\tilde{\mu}_{T}$. Here we have defined

$$
\begin{aligned}
\epsilon_{1} & \equiv \sqrt{\tilde{\Delta}^{2}-4 \tilde{\mu}_{N}} \\
\epsilon_{2} & \equiv \frac{2 D-\tilde{\mu}_{N}-\tilde{\mu}_{T}}{\sqrt{\left|D-\tilde{\mu}_{T}\right|}} \\
\epsilon_{3} & \equiv \frac{\sqrt{D-\tilde{\mu}_{N}}}{\sqrt{\left|D-\tilde{\mu}_{T}\right|}}
\end{aligned}
$$

and

$$
\cos \gamma^{\prime} \equiv \sqrt{1-1 / \epsilon_{1}^{2}}, \quad \sin \gamma^{\prime} \equiv 1 / \epsilon_{1}
$$

In the large curvature limit $(R \rightarrow \infty)$ where $\gamma \rightarrow \pi / 2$ and $\gamma^{\prime} \rightarrow 0$, the coefficients are simplified to

$$
\begin{aligned}
h_{N \nu}^{ \pm} & =\epsilon_{1}\left[\cos 2 \pi f-(-1)^{\nu} \cos (\delta \varphi-2 \pi f)\right] \\
h_{T \nu}^{ \pm} & =\epsilon_{1}-(-1)^{\nu} \epsilon_{2} \quad\left(\tilde{\mu}_{T}<D\right) \\
\sum_{\nu} h_{T \nu}^{ \pm} & =2 \epsilon_{1} \cos k_{r} L_{T}+2 \epsilon_{2} \sin k_{r} L_{T} \quad\left(\tilde{\mu}_{T}>D\right)
\end{aligned}
$$

and

$$
\begin{aligned}
& N_{a}=N_{0}+\frac{\sqrt{D}}{-\tilde{\mu}_{N}} \cos (4 \pi f-\delta \varphi), \\
& N_{b}=N_{0}+\frac{\sqrt{D}}{-\tilde{\mu}_{N}} \cos \delta \varphi
\end{aligned}
$$

with

$$
N_{0} \equiv \frac{\sqrt{D-\tilde{\mu}_{N}}}{-\tilde{\mu}_{N}}+\frac{\sqrt{D}+\sqrt{D-\tilde{\mu}_{N}}}{\tilde{\mu}_{T}}+\frac{\tilde{\mu}_{T}-\tilde{\mu}_{N}}{2 \tilde{\mu}_{T} \sqrt{D}}
$$

where $N_{0}$ is the value of the normalization constants $N_{a, b}$ averaged over the phases.
1 P. A. M. Dirac, Proc. R. Soc. (London) A 117, 610 (1928).

2 P. A. M. Dirac, Proc. R. Soc. (London) A 126, 360 (1930).

3 E. Majorana, Nuovo Cimento. 14, 171 (1937).

4 A. Yu. Kitaev, Ann. Phys. 303, 2 (2003).

5 S. Das Sarma, M. Freedman, and C. Nayak, Physics Today 59, 32 (2006).

${ }^{6}$ C. Nayak, S. H. Simon, A. Stern, M. Freedman, and S. Das Sarma, Rev. Mod. Phys. 80, 1083 (2008).
7 F. Wilczek, Nat. Phys. 5, 614 (2009).

${ }^{8}$ M. Franz, Physics 3, 24 (2010).

9 A. Yu Kitaev, Physics-Uspekhi 44, 131 (2001).

10 J. D. Sau, R. M. Lutchyn, S. Tewari, and S. Das Sarma, Phys. Rev. Lett. 104, 040502 (2010).

11 R. M. Lutchyn, J. D. Sau, and S. Das Sarma, Phys. Rev. Lett. 105, 077001 (2010).

12 J. Alicea, Phys. Rev. B 81, 125318 (2010). 
13 Y. Oreg, G. Refael, and F. von Oppen, Phys. Rev. Lett. 105, 177002 (2010).

14 J. Alicea, Y. Oreg, G. Refael, F. von Oppen, and M. P. A. Fisher, Nat. Phys. 7, 412 (2011).

15 J. Alicea, Rep. Prog. Phys. 75, 076501 (2012).

${ }^{16}$ C. W. J. Beenakker, Annual Review of Condensed Matter Physics 4, 113 (2013).

17 V. Mourik, K. Zuo, S. M. Frolov, S. R. Plissard, E. P. A. M. Bakkers, and L. P. Kouwenhoven, Science 336, 1003 (2012).

18 M. T. Deng, C. L. Yu, G. Y. Huang, M. Larsson, P. Caroff, and H. Q. Xu, Nano Letters 12, 6414 (2012).

19 A. Das, Y. Ronen, Y. Most, Y. Oreg, M. Heiblum, and H. Shtrikman, Nat. Phys. 8, 12 (2012).

${ }^{20}$ J. Liu, A. C. Potter, K. T. Law, and P. A. Lee, Phys. Rev. Lett. 109, 267002 (2012).

21 F. Pientka, G. Kells, A. Romito, P. W. Brouwer, and F. von Oppen, Phys. Rev. Lett. 109, 227006 (2012).

22 D. Rainis, L. Trifunovic, J. Klinovaja, D. Loss, Phys. Rev. B 87, 024515 (2013).

${ }^{23}$ L. Jiang, D. Pekker, J. Alicea, G. Refael, Y. Oreg, and F. von Oppen, Phys. Rev. Lett. 107, 236401 (2011).

${ }^{24}$ B. van Heck, A. R. Akhmerov, F. Hassler, M. Burrello, and C. W.
J. Beenakker, New J. of Phys. 14, 035019 (2012).

${ }^{25}$ F. Pientka, A. Romito, M. Duckheim, Y. Oreg, and F. von Oppen, New J. of Phys. 15, 025001 (2013).

${ }^{26}$ L. Fu and C. L. Kane, Phys. Rev. B 79, 161408(R) (2009).

27 S.-Q. Shen, Topological Insulators, vol. 174 of Springer Series in Solid-State Sciences (Springer, Berlin, 2012).

28 J. Splettstoesser, M. Governale, and U. Zülicke, Phys. Rev. B 68, 165341 (2003).

${ }^{29}$ Even though we assume a circular ring for convenience, it need not be a perfect circle as long as the nanowire forms a closed loop. Essential physics does not depend on the specific geometry. Experimental realization may prefer a closed loop with straight semiconductor segments (instead of curved segments) in favor of easier layering of nanowires and superconductors.

${ }^{30}$ In the closed ring geometry, the applied magnetic flux $f \neq 0$ adds additional relative rotation between the polarization axes at two ends of the same region.

${ }^{31}$ For the supercurrent, in general, one has to calculate all the states including the (almost) continuum of the bulk states. In our study we focus on the contribution from the subgap states. 